\title{
Constraining type II 2HDM in light of LHC Higgs searches
}

\section{Baradhwaj Coleppa, Felix Kling and Shufang Su}

Department of Physics, University of Arizona

1118 E. 4th st., Tucson, AZ 85721, U.S.A.

E-mail: baradhwa@email.arizona.edu, kling@email.arizona.edu, shufang@email.arizona.edu

Abstract: We study the implication of the LHC Higgs search results on the Type II Two Higgs-Doublet Model. In particular, we explore the scenarios in which the observed $126 \mathrm{GeV}$ Higgs signal is interpreted as either the light CP-even Higgs $h^{0}$ or the heavy CP-even Higgs $H^{0}$. Imposing both theoretical and experimental constraints, we analyze the surviving parameter regions in $m_{H}\left(m_{h}\right), m_{A}, m_{H^{ \pm}}, \tan \beta$ and $\sin (\beta-\alpha)$. We further identify the regions that could accommodate a $126 \mathrm{GeV}$ Higgs with cross sections consistent with the observed Higgs signal. We find that in the $h^{0}-126$ case, we are restricted to narrow regions of $\sin (\beta-\alpha) \approx \pm 1$ with $\tan \beta$ up to 4 , or an extended region with $0.55<$ $\sin (\beta-\alpha)<0.9$ and $1.5<\tan \beta<4$. The values of $m_{H}, m_{A}$ and $m_{H^{ \pm}}$, however, are relatively unconstrained. In the $H^{0}-126$ case, we are restricted to a narrow region of $\sin (\beta-\alpha) \sim 0$ with $\tan \beta$ up to about 8 , or an extended region of $\sin (\beta-\alpha)$ between -0.8 to -0.05 , with $\tan \beta$ extended to 30 or higher. $m_{A}$ and $m_{H^{ \pm}}$are nearly degenerate due to $\Delta \rho$ constraints. Imposing flavor constraints shrinks the surviving parameter space significantly for the $H^{0}-126$ case, limiting $\tan \beta \lesssim 10$, but has little effect in the $h^{0}-126$ case. We also investigate the correlation between $\gamma \gamma, V V$ and $b b / \tau \tau$ channels. $\gamma \gamma$ and $V V$ channels are most likely to be highly correlated with $\gamma \gamma: V V \sim 1$ for the normalized cross sections.

KEYwords: Phenomenological Models

ARXIV EPRINT: 1305.0002 


\section{Contents}

1 Introduction 1

2 Type II 2HDM 3

2.1 Potential, masses and mixing angles 3

3 Constraints and analyses $\quad 4$

3.1 Theoretical and experimental constraints 4

3.2 Analysis method 6

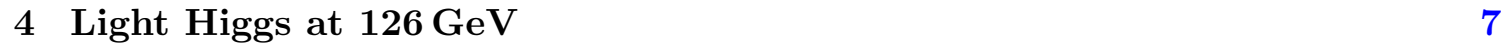

$\begin{array}{lll}4.1 & \text { Cross sections and correlations } & 7\end{array}$

$\begin{array}{lll}4.2 & \text { Parameter spaces } & 11\end{array}$

5 Heavy Higgs at $126 \mathrm{GeV} \quad 16$

$\begin{array}{lll}5.1 & \text { Cross sections and correlations } & 16\end{array}$

$\begin{array}{lll}5.2 & \text { Parameter spaces } & 19\end{array}$

6 Other Higgs channels 22

$\begin{array}{lll}7 & \text { Conclusions } & 25\end{array}$

\section{Introduction}

The discovery of a resonance at $126 \mathrm{GeV}$ with properties consistent with the Standard Model (SM) Higgs boson in both the ATLAS [1, 2] and CMS experiments [3, 4] is undoubtedly the most significant experimental triumph of the Large Hadron Collider (LHC) to date. The nature of this particle, as regards its $\mathrm{CP}$ properties and couplings, are currently being established [4-7]. Though further data would undoubtedly point us in the right direction, at this point it is useful to explore the implication of the current Higgs search results on models beyond the SM. There are quite a few models that admit a scalar particle in their spectrum and many of them can have couplings and decays consistent with the SM Higgs boson. Thus it behooves us to constrain these models as much as possible with the Higgs search results at hand.

One of the simplest extensions of the SM involves enlarged Higgs sectors. This can be done by simply adding more scalar doublets, or considering Higgs sectors with more complicated representations. In the work, we will study the Two Higgs-Doublet Models $(2 \mathrm{HDM})$ that involve two scalar doublets both charged under the $\mathrm{SM} \mathrm{SU}(2)_{L} \times \mathrm{U}(1)_{Y}$ gauge symmetries [8-11]. The neutral components of both the Higgs fields develop vacuum 
expectation values (vev), breaking $\mathrm{SU}(2)_{L} \times \mathrm{U}(1)_{Y}$ down to $\mathrm{U}(1)_{\mathrm{em}}$. Assuming no CPviolation in the Higgs sector, the resulting physical spectrum for the scalars is enlarged relative to the SM and includes light and heavy neutral CP-even Higgses $\left(h^{0}\right.$ and $\left.H^{0}\right)$, charged Higgses $\left(H^{ \pm}\right)$, and a pseudoscalar $A^{0}$. In addition to the masses, two additional parameters are introduced in the theory: the ratio of the vevs of the two Higgs fields $(\tan \beta)$, and the mixing of the two neutral CP-even Higgses $(\sin \alpha)$.

There are many types of $2 \mathrm{HDM}$, each differing in the way the two Higgs doublets couple to the fermions (for a comprehensive review, see [8]). In this work, we will be concentrating on the Type II case, in which one Higgs doublet couples to the up-type quarks, while the other Higgs doublet couples to the down-type quarks and leptons. This model is of particular interest as it shares many of the features of the Higgs sector of the Minimal Supersymmetric Standard Model (MSSM). This enables us to translate existing LHC MSSM results to this case. Before proceeding, we point out that over the last few months, there have been various studies on the $2 \mathrm{HDM}$ based on the recent discovery [12-25]. While most studies concentrated on finding regions of parameter space that admit $\sigma \times \mathrm{Br}$ values reported by the LHC experiments in various channels, some also looked at correlations between the various decay channels. The authors of ref. [12] and ref. [13] did the initial study of looking at the $\tan \beta-\sin \alpha$ plane where the observed Higgs signal is feasible, interpreting the discovered scalar as either the light or the heavy CP-even Higgs boson. Ref. [14-19] fit the observed Higgs signals in various $2 \mathrm{HDM}$ scenarios, taken into account theoretical and experimental constraints. Ref. [20] also paid careful attention to various Higgs production modes. Ref. [21] focused on the CP-violating Type II 2HDM. Ref. [22] studied the case of nearly degenerate Higgs bosons. In addition, ref. [23, 24] investigated the possibility that the signal could correspond to the pseudoscalar $A^{0}$ - in this context, it is worth remarking that ref. [26] considered the pseudoscalar interpretation of the observed $126 \mathrm{GeV}$ resonance and found that while it is strongly disfavored, the possibility is not yet ruled out at the $5 \sigma$ level. $^{1}$

In the present paper, we extended the above analyses by combining all the known experimental constraints (the LEP, Tevatron and the LHC Higgs search bounds, and precision observables) with the theoretical ones (perturbativity, unitarity, and vacuum stability), as well as flavor constraints. A unique aspect of the present work is that our analysis looks at combinations of all parameters of the theory to identify regions that survive all the theoretical and experimental constraints. We further focus on regions that could accommodate the observed Higgs signal as either the light or the heavy CP-even Higgs, and are thus interesting from a collider study perspective. This enables us to draw conclusions about correlations between different masses and mixing angles to help identify aspects of the model that warrant future study.

We start by briefly introducing the structure and parameters of the Type II 2HDM in section 2. In section 3, we discuss the theoretical constraints and experimental bounds, and outline our analysis methodology. In section 4, we present our results for the light CPeven Higgs being the observed $126 \mathrm{GeV}$ SM-like Higgs boson, looking at surviving regions

\footnotetext{
${ }^{1}$ The latest experimental results indicate that the pseudoscalar interpretation of the $126 \mathrm{GeV}$ excess is disfavored [4-6].
} 
in various combinations of free parameters. In section 5 , we do the same for the heavy CP-even Higgs as the observed $126 \mathrm{GeV}$ SM-like Higgs boson. In section 6, we explore the implications for the Vector Boson Fusion (VBF) or $V H$ associated production, and decays of Higgs into $b b$ and $\tau \tau$ channels. We conclude in section 7 .

\section{Type II 2HDM}

In this section, we briefly describe the Type II $2 \mathrm{HDM}$, focusing on the particle content, Higgs couplings, and model parameters. For more details about the model, see ref. [8] for a recent review of the theory and phenomenology of $2 \mathrm{HDM}$.

\subsection{Potential, masses and mixing angles}

Labeling the two $\mathrm{SU}(2)_{L}$ doublet scalar fields $\Phi_{1}$ and $\Phi_{2}$, the most general potential for the Higgs sector can be written down in the following form:

$$
\begin{aligned}
V\left(\Phi_{1}, \Phi_{2}\right)= & m_{11}^{2} \Phi_{1}^{\dagger} \Phi_{1}+m_{22}^{2} \Phi_{2}^{\dagger} \Phi_{2}-m_{12}^{2}\left(\Phi_{1}^{\dagger} \Phi_{2}+\text { h.c. }\right) \\
& +\frac{1}{2} \lambda_{1}\left(\Phi_{1}^{\dagger} \Phi_{1}\right)^{2}+\frac{1}{2} \lambda_{2}\left(\Phi_{2}^{\dagger} \Phi_{2}\right)^{2}+\lambda_{3}\left(\Phi_{1}^{\dagger} \Phi_{1}\right)\left(\Phi_{2}^{\dagger} \Phi_{2}\right)+\lambda_{4}\left(\Phi_{1}^{\dagger} \Phi_{2}\right)\left(\Phi_{2}^{\dagger} \Phi_{1}\right) \\
& +\frac{1}{2}\left\{\lambda_{5}\left(\Phi_{1}^{\dagger} \Phi_{2}\right)^{2}+\text { h.c. }\right\}+\left\{\left[\lambda_{6}\left(\Phi_{1}^{\dagger} \Phi_{1}\right)+\lambda_{7}\left(\Phi_{2}^{\dagger} \Phi_{2}\right)\right]\left(\Phi_{1}^{\dagger} \Phi_{2}\right)+\text { h.c. }\right\} .
\end{aligned}
$$

We impose a discrete $Z_{2}$ symmetry on the Lagrangian, the effect of which is to render $m_{12}, \lambda_{6}, \lambda_{7}=0 .^{2}$ Note that one consequence of requiring $m_{12}=0$ is that there is no so called decoupling limit in which only one SM-like Higgs appears at low energy while all other Higgses are heavy and decoupled from the low energy spectrum. After electroweak symmetry breaking (EWSB): $\left\langle\phi_{1}^{0}\right\rangle=v_{1} / \sqrt{2},\left\langle\phi_{2}^{0}\right\rangle=v_{2} / \sqrt{2}$ with $\sqrt{v_{1}^{2}+v_{2}^{2}}=246 \mathrm{GeV}$, we are left with six free parameters, which can be chosen as the four Higgs masses $\left(m_{h}, m_{H}\right.$, $\left.m_{A}, m_{H^{ \pm}}\right)$, a mixing angle $\sin \alpha$ between the two CP-even Higgses, and the ratio of the two vacuum expectation values, $\tan \beta=v_{2} / v_{1}$.

Writing the two Higgs fields as:

$$
\Phi_{i}=\left(\begin{array}{c}
\phi_{i}^{+} \\
\left(v_{i}+\phi_{i}^{0}+i G_{i}\right) / \sqrt{2}
\end{array}\right),
$$

the mass eigenstates of the physical scalars can be written as:

$$
\left(\begin{array}{c}
H^{0} \\
h^{0}
\end{array}\right)=\left(\begin{array}{cc}
\cos \alpha & \sin \alpha \\
-\sin \alpha & \cos \alpha
\end{array}\right)\left(\begin{array}{l}
\phi_{1}^{0} \\
\phi_{2}^{0}
\end{array}\right), \quad \begin{aligned}
& A^{0}=-G_{1} \sin \beta+G_{2} \cos \beta \\
& H^{ \pm}=-\phi_{1}^{ \pm} \sin \beta+\phi_{2}^{ \pm} \cos \beta
\end{aligned} .
$$

For our purposes, it is useful to express the quartic couplings $\lambda_{1 \ldots 5}$ in terms of the physical Higgs masses, $\tan \beta$ and the mixing angle $\alpha$ :

$$
\begin{aligned}
& \lambda_{1}=\frac{m_{H}^{2} \cos ^{2} \alpha+m_{h}^{2} \sin ^{2} \alpha}{v^{2} \cos ^{2} \beta}, \quad \lambda_{2}=\frac{m_{H}^{2} \sin ^{2} \alpha+m_{h}^{2} \cos ^{2} \alpha}{v^{2} \cos ^{2} \beta} \\
& \lambda_{3}=\frac{\sin 2 \alpha\left(m_{H}^{2}-m_{h}^{2}\right)+2 \sin 2 \beta m_{H^{ \pm}}^{2}}{v^{2} \sin 2 \beta}, \quad \lambda_{4}=\frac{m_{A}^{2}-2 m_{H^{ \pm}}^{2}}{v^{2}}, \quad \lambda_{5}=-\frac{m_{A}^{2}}{v^{2}} .
\end{aligned}
$$

\footnotetext{
${ }^{2}$ Ref. [15], which also addresses similar issues as in this paper, allowed for a soft breaking of the $Z_{2}$ symmetry with $m_{12}^{2} \neq 0$. In this paper, we don't consider such soft-breaking terms.
} 


\begin{tabular}{|l|l|l|l|l|l|}
\hline$\xi_{h}^{V V}$ & $\sin (\beta-\alpha)$ & $\xi_{H}^{V V}$ & $\cos (\beta-\alpha)$ & $\xi_{A}^{V V}$ & 0 \\
\hline$\xi_{h}^{u}$ & $\cos \alpha / \sin \beta$ & $\xi_{H}^{u}$ & $\sin \alpha / \sin \beta$ & $\xi_{A}^{u}$ & $\cot \beta$ \\
\hline$\xi_{h}^{d, l}$ & $-\sin \alpha / \cos \beta$ & $\xi_{H}^{d, l}$ & $\cos \alpha / \cos \beta$ & $\xi_{A}^{d, l}$ & $\tan \beta$ \\
\hline
\end{tabular}

Table 1. The multiplicative factor $\xi$ by which the couplings of the CP-even Higgses and the CPodd Higgs to the gauge bosons and fermions scale with respect to the SM value. The superscripts $u, d, l$ and $V V$ refer to the up-type quarks, down-type quarks, leptons, and $W W / Z Z$ respectively.

Imposing the perturbativity and unitarity bounds, as explained below in section 3.1, typically leads to an upper bound on the masses of $H^{0}, A^{0}$ and $H^{ \pm}$. The couplings of the $\mathrm{CP}$-even Higgses and CP-odd Higgs to the SM gauge bosons and fermions are scaled by a factor $\xi$ relative to the SM value - these are presented in table 1. In order to translate the ATLAS and CMS limits, we need to pay particular attention to the couplings of the light (heavy) CP-even Higgs to the SM gauge bosons (controlling the partial decay width to $W W, Z Z$ as well as $\gamma \gamma$ channels) and to the top quark (controlling the gluon fusion production cross section), as well as to the bottom quark (controlling the $b b$ partial decay width, which enters the total decay width as well). From table 1 , we see that the relevant couplings are proportional to $\sin (\beta-\alpha)(\cos (\beta-\alpha)), 1 / \sin \beta$ and $1 / \cos \beta$. Thus, even though it is customary to look at the combination of parameters $(\sin \alpha, \tan \beta)$, we present our results in section 4 and 5 using $\sin (\beta-\alpha)$ and $\tan \beta$ as the independent parameters (in addition to the masses of the physical Higgses) to manifest the effects on the Higgs couplings to gauge bosons. Using $\sin (\beta-\alpha)$ instead of $\sin \alpha$ has the additional advantage of being basis-independent, as explained in ref. [27-29].

\section{Constraints and analyses}

\subsection{Theoretical and experimental constraints}

To implement the various experimental and theoretical constraints, we have employed two programs: the 2HDM Calculator (2HDMC) [30] to calculate the Higgs couplings, compute all the decay branching fractions of the Higgses, and implement all the theoretical constraints; and HiggsBounds 3.8 [31] to consistently put in all the experimental constraints on the model. Here, we briefly describe the list of theoretical and experimental bounds that are of interest.

\section{Theoretical constraints:}

- Vacuum Stability: this implies that the potential should be bounded from below, which is translated to various conditions for the quartic couplings in the Higgs potential [36-38]: $\lambda_{1}>0, \lambda_{2}>0, \lambda_{3}>-\sqrt{\lambda_{1} \lambda_{2}}$, and $\lambda_{3}+\lambda_{4}-\left|\lambda_{5}\right|>-\sqrt{\lambda_{1} \lambda_{2}}$. With eqs. (2.4) and (2.5), the above requirements serve to constrain the Higgs masses and angles.

- Perturbativity: 2HDMC imposes constraints on the physical Higgs quartic couplings, specifically demanding that $\lambda_{h_{i} h_{j} h_{k} h_{l}}<4 \pi$ to stay inside the perturbative regime. 
Note that even though these are different from the $\lambda \mathrm{s}$ in the Higgs potential in eq. (2.1), we can still use eqs. (2.4) and (2.5) as rough guides to understand the perturbative bounds, as we will do in later sections to explain the features of our results. The top yukawa coupling $y_{t}$ could also become nonperturbative for very small $\tan \beta$. We require the perturbativity of $y_{t}$ at scales below $1 \mathrm{TeV}$, which results in $\tan \beta \gtrsim 0.35[39]$.

- Unitarity: it is well known that in the SM, the scattering cross section for the longitudinal $W$ modes is unitary only if the Higgs exchange diagrams are included. Since the couplings of the Higgs are modified in the $2 \mathrm{HDM}$, we need to ensure unitarity by demanding that the $S$ matrix of all scattering cross sections of Higgs-Higgs and Higgs $-V_{L}$ (where $V_{L}$ is either $W_{L}$ or $Z_{L}$ ) have eigenvalues bounded by $16 \pi$ [40].

Experimental constraints: the LHC experiments have searched for the SM Higgs in $\gamma \gamma$, $Z Z, W W, \tau \tau$ and $b b$ channels. Both the ATLAS and CMS collaboration have reported the observation of a new resonance at a mass of around $126 \mathrm{GeV}$ with more than $5 \sigma$ significance $[1-7,41-52]$. The production cross sections and partial decay widths of the 2HDM Higgses to the various SM final states differ from that of the SM Higgs, which can be obtained using the coupling scaling factors $\xi$ from table 1 . Thus, we can identify the regions in parameter space where the signal cross sections are compatible with the Higgs signal observed at the ATLAS and CMS collaborations. We can also translate the exclusion bounds on the Higgs search to the ones in the 2HDM. We used HiggsBounds 3.8 to impose the exclusion limits from Higgs searches at the LEP and the Tevatron [53-57]. We also incorporated the latest Higgs search results at the LHC [2, 4, 41-52, 58-64].

$Z$-pole precision observables, in particular, the oblique parameters $S, T$ (or equivalently, $\Delta \rho$, which is the deviation of $\rho \equiv \frac{m_{W}^{2}}{m_{Z}^{2} \cos ^{2} \theta_{W}}$ from the SM value), and $U$ [65] constrain any new physics model that couples to the $W$ and $Z$. In particular, $T$ imposes a strong constraint on the amount of custodial symmetry breaking in the new physics sector. In the case of $2 \mathrm{HDM}$, the mass difference between the various Higgses are therefore highly constrained [66], which leads to interesting correlations between some of the masses, as will be demonstrated in section 4 and section 5 . In our analysis, we require the contribution from extra Higgses to $S$ and $T$ to fall within the $90 \%$ C.L. $S-T$ contour, for a SM Higgs reference mass of $126 \mathrm{GeV}$ [67]. In addition, the charged Higgs contributes to $Z b b$ coupling [68], which has been measured precisely at the LEP via the observable $R_{b}=\Gamma(Z \rightarrow b \bar{b}) / \Gamma(Z \rightarrow$ hadrons $)$ [69]. Imposing bounds from $R_{b}$ rules out small $\tan \beta$ regions for a light charged Higgs.

We also show the effect on the available parameter spaces once bounds from flavor sector are imposed in addition to the ones described. To do this, we employed the program SuperIso 3.3 [70], which incorporates, among other things, bounds from $B \rightarrow X_{s} \gamma, \Delta M_{B_{d}}$, $B^{-} \rightarrow \tau^{-} \bar{\nu}_{\tau}, D_{s}^{ \pm} \rightarrow \tau^{ \pm}\left(\mu^{ \pm}\right) \nu, B \rightarrow \tau^{+} \tau^{-}$and $B_{d, s} \rightarrow \mu^{+} \mu^{-}$[71-77]. A summary of flavor bounds can be found in ref. [78]. We have used the latest bounds either from PDG [71] ${ }^{3}$ or from individual experiment. To show the impact of the flavor constraints on the 2HDM parameter space, in figure 1, we present the regions excluded by various flavor constraints in

\footnotetext{
${ }^{3}$ And 2013 partial update for the 2014 edition.
} 

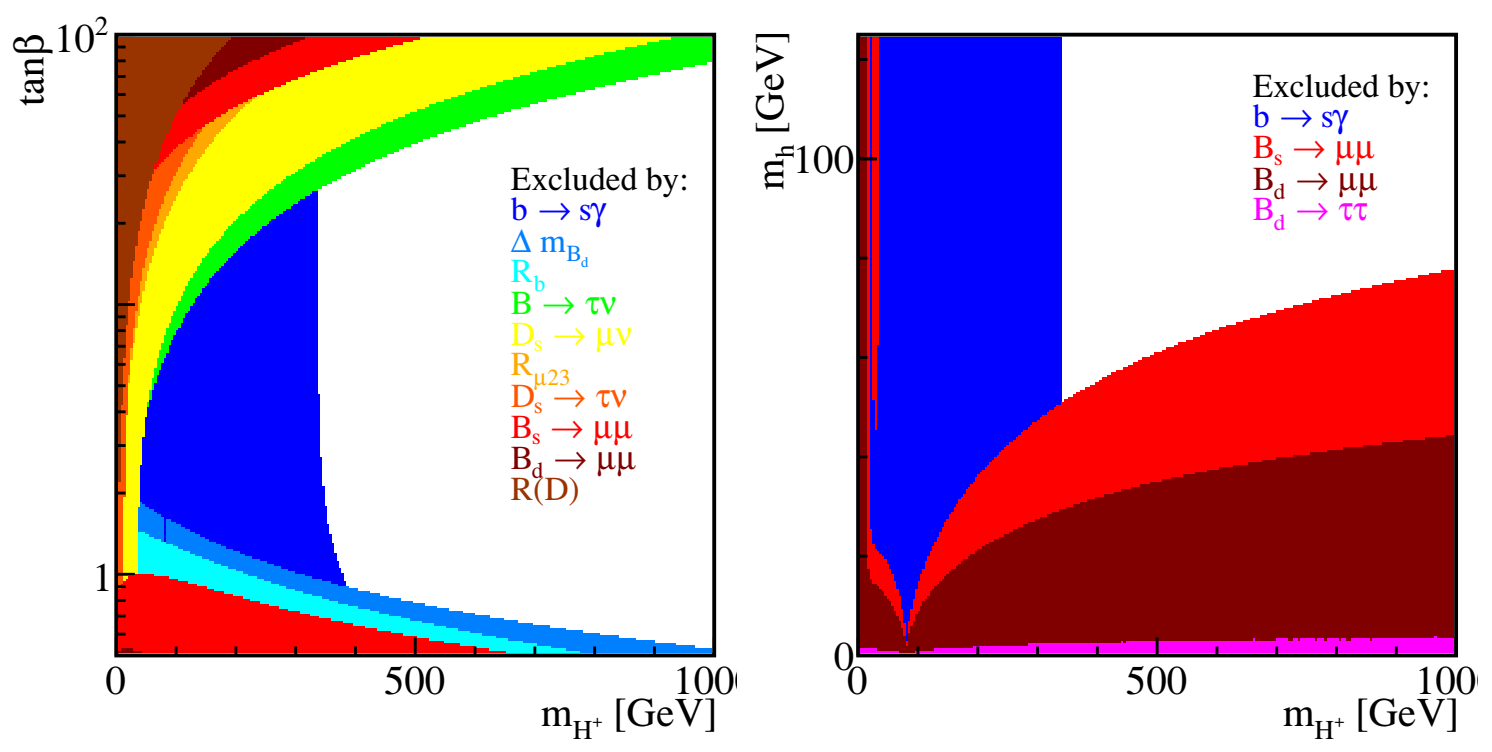

Figure 1. Regions of parameter space excluded by various flavor constraints. The left plot shows the $m_{H^{ \pm}}$versus $\tan \beta$ plane for fixed $m_{h}=125 \mathrm{GeV}, m_{H}=400 \mathrm{GeV}, m_{A}=200 \mathrm{GeV}$ and $\sin (\beta-$ $\alpha)=-0.1$. The right plot shows the $m_{H^{ \pm}}$versus $m_{h}$ plane for $m_{A}=m_{H^{ \pm}}, m_{H}=125 \mathrm{GeV}$, $\tan \beta=5$ and $\sin (\beta-\alpha)=-0.01$.

the $m_{H^{ \pm}}$versus $\tan \beta$ plane (left panel) and the $m_{H^{ \pm}}$versus $m_{h}$ plane (right panel). While $B \rightarrow X_{s} \gamma$ excludes $m_{H^{ \pm}}$up to about $300 \mathrm{GeV}$ for all $\tan \beta, B^{-} \rightarrow \tau^{-} \bar{\nu}_{\tau}$ and $\Delta M_{B_{d}}$ provide the strongest constraints at large and small $\tan \beta$, respectively. The strongest bound on the neutral Higgs mass comes from $B_{s} \rightarrow \mu^{+} \mu^{-}$, which excludes $m_{h}$ at about $50 \mathrm{GeV}$ or lower.

In addition, we included the latest results from BaBar on $\bar{B} \rightarrow D \tau \bar{\nu}_{\tau}$ and $\bar{B} \rightarrow$ $D^{*} \tau \bar{\nu}_{\tau}$ [79], which observed excesses over the SM prediction at about $2 \sigma$ level. We treat the observed excesses as upper bounds and take the $95 \%$ C.L. range as $R(D)<0.58$ and $R\left(D^{*}\right)<0.39$. Note that as pointed out in ref. [79], the excesses in both $R(D)$ and $R\left(D^{*}\right)$ can not be simultaneously explained by the Type II 2HDM [80, 81]. Other new physics contributions have to enter if the excesses in both $R(D)$ and $R\left(D^{*}\right)$ stay in the future. Flavor constraints on the Higgs sector are, however, typically more modeldependent. Therefore, our focus in this work is mainly on the implication of the Higgs search results on the Type II $2 \mathrm{HDM}$, and we only impose the flavor bounds at the last step to indicate how the surviving regions further shrink.

\subsection{Analysis method}

In our analysis, we considered two scenarios:

- $h^{0}-126$ case where $m_{h}=126 \mathrm{GeV}$ with $m_{H}>126 \mathrm{GeV}$,

- $H^{0}-126$ case where $m_{H}=126 \mathrm{GeV}$ with $m_{h}<126 \mathrm{GeV}$ 
and scanned over the entire remaining parameter space varying $m_{H}\left(\right.$ or $\left.m_{h}\right), m_{A}, m_{H^{ \pm}}$, $\tan \beta$ and $\sin (\beta-\alpha)$ :

$$
\begin{aligned}
& 20 \mathrm{GeV} \leq m_{A}, m_{H^{ \pm}} \leq 900 \mathrm{GeV} \quad \text { in steps of } 20 \mathrm{GeV}, \\
& -1 \leq \sin (\beta-\alpha) \leq 1 \quad \text { in steps of } 0.05, \\
& \mathbf{h}^{\mathbf{0}}-\mathbf{1 2 6} \text { case }: 0.25 \leq \tan \beta \quad \leq 5 \\
& 126 \mathrm{GeV} \leq m_{H} \quad \leq 900 \mathrm{GeV} \quad \text { in steps of } 20 \mathrm{GeV}, \\
& \mathbf{H}^{\mathbf{0}}-\mathbf{1 2 6} \text { case }: 1 \leq \tan \beta \quad \leq 30 \quad \text { in steps of } 1 \text {, } \\
& 6 \mathrm{GeV} \leq m_{h} \quad<126 \mathrm{GeV} \quad \text { in steps of } 5 \mathrm{GeV} .
\end{aligned}
$$

In certain regions in which very few points are left after all the constraints are imposed, we generated more points with smaller steps. We used the 2HDMC 1.2beta [30] which tested if each parameter point fulfills the theoretical and experimental constraints implemented in HiggsBounds 3.8 [31]. New LHC results that are not included in HiggsBounds 3.8 were implemented in addition. In particular, the CMS results on MSSM Higgs search in $\tau \tau$ channel [61-64] were imposed using the cross section limits reverse-engineered from bounds in $m_{A}-\tan \beta$ plane for $m_{h}^{\max }$ scenario, as provided in HiggsBounds 4.0 [31]. We also required each parameter point to satisfy the precision constraints, in particular, $S$ and $T$, as well as $R_{b}$.

We further required either $h^{0}$ or $H^{0}$ to satisfy the dominant gluon fusion cross section requirement for $\gamma \gamma, W W$ and $Z Z$ channels to accommodate the observed Higgs signal at $95 \%$ C.L. $[4,7]$ :

$$
0.7<\frac{\sigma\left(g g \rightarrow h^{0} / H^{0} \rightarrow \gamma \gamma\right)}{\sigma_{\mathrm{SM}}}<1.5, \quad 0.6<\frac{\sigma\left(g g \rightarrow h^{0} / H^{0} \rightarrow W W / Z Z\right)}{\sigma_{\mathrm{SM}}}<1.3,
$$

in which we have taken the tighter limits from the ATLAS and CMS results, as well as the tighter results for the $W W$ and $Z Z$ channel. In the last step, we imposed the flavor bounds on all points that satisfy eq. (3.7) using the SuperIso 3.3 program to study the consequence of the flavor constraints.

\section{Light Higgs at $126 \mathrm{GeV}$}

\subsection{Cross sections and correlations}

Before presenting the results of the numerical scanning of parameter regions with all the theoretical and experimental constraints imposed, let us first study the $\tan \beta$ and $\sin (\beta-\alpha)$ dependence of the cross sections for the major search channels at the LHC: $g g \rightarrow h^{0} \rightarrow \gamma \gamma, W W / Z Z$. Both production cross sections and decay branching fractions are modified relative to the SM values:

$$
\frac{\sigma \times \operatorname{Br}\left(g g \rightarrow h^{0} \rightarrow X X\right)}{\mathrm{SM}}=\frac{\sigma\left(g g \rightarrow h^{0}\right)}{\sigma_{\mathrm{SM}}} \times \frac{\operatorname{Br}\left(h^{0} \rightarrow X X\right)}{\operatorname{Br}\left(h_{\mathrm{SM}} \rightarrow X X\right)},
$$

for $X X=\gamma \gamma, V V$. Note that since the $W W$ and $Z Z$ couplings are modified the same way in the Type II 2HDM, we use $V V$ to denote both $W W$ and $Z Z$ channels. 

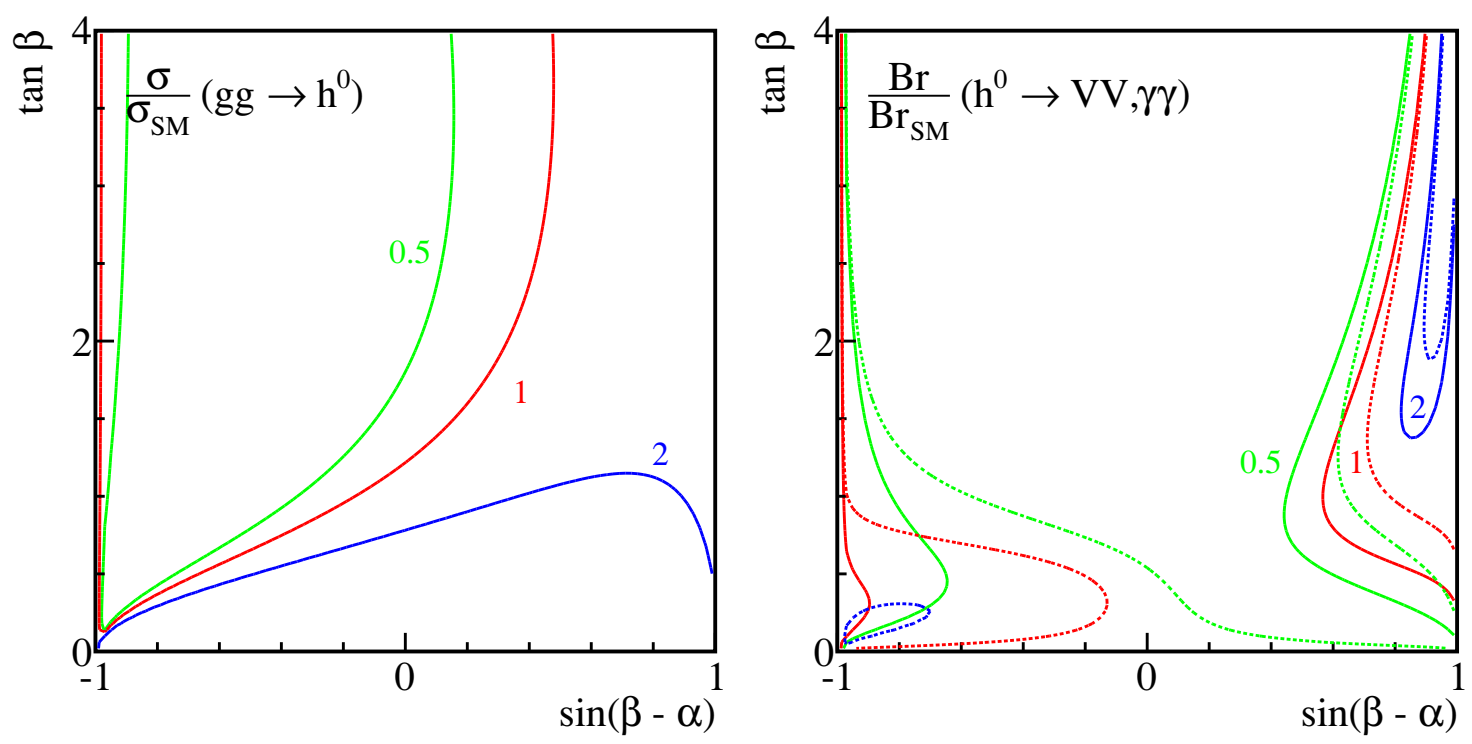

Figure 2. The normalized $g g \rightarrow h^{0}$ production cross section contours (left panel) and $h^{0} \rightarrow V V$ (solid lines of the right panel) and $h^{0} \rightarrow \gamma \gamma$ (dashed lines of the right panel) branching fractions in the $h^{0}-126$ case. The contour lines are $\sigma / \sigma_{\mathrm{SM}}, \mathrm{Br} / \mathrm{Br}_{\mathrm{SM}}=0.5$ (green), 1 (red), and 2 (blue).

The ratio of the gluon fusion cross section normalized to the SM value can be written as:

$$
\begin{aligned}
& \frac{\sigma\left(g g \rightarrow h^{0}\right)}{\sigma_{\mathrm{SM}}}=\frac{\cos ^{2} \alpha}{\sin ^{2} \beta}+\frac{\sin ^{2} \alpha}{\cos ^{2} \beta} \frac{\left|A_{1 / 2}\left(\tau_{b}\right)\right|^{2}}{\left|A_{1 / 2}\left(\tau_{t}\right)\right|^{2}} \\
& =\left[\frac{\cos (\beta-\alpha)}{\tan \beta}+\sin (\beta-\alpha)\right]^{2}+[\cos (\beta-\alpha) \tan \beta-\sin (\beta-\alpha)]^{2} \frac{\left|A_{1 / 2}\left(\tau_{b}\right)\right|^{2}}{\left|A_{1 / 2}\left(\tau_{t}\right)\right|^{2}} .
\end{aligned}
$$

The expression for the fermion loop functions $A_{1 / 2}\left(\tau_{t, b}\right)$ can be found in ref. [66]. The first term in eq. (4.2) is the top-loop contribution, and the second term is the bottom-loop contribution. In the SM, the top-loop contributes dominantly to the gluon fusion diagram, while the bottom-loop contribution is negligibly small. The situation alters in type II $2 \mathrm{HDM}$ for large $\tan \beta$, when the bottom-loop contribution can be substantial due to the enhanced bottom Yukawa [12]. We also rewrite it in $\sin (\beta-\alpha), \cos (\beta-\alpha)$ and $\tan \beta$ in eq. (4.3) to make their dependence explicit.

In the left panel of figure 2, we show contours of $\sigma / \sigma_{\mathrm{SM}}$ for the gluon fusion: $\sigma / \sigma_{\mathrm{SM}}=$ 0.5 (green), 1 (red), and 2 (blue). While contours of $\sigma / \sigma_{\mathrm{SM}} \geq 1$ accumulate in $\sin (\beta-\alpha) \sim$ -1 region, there is a wide spread of the contours for $\sin (\beta-\alpha)>0$. For most regions of $\sin (\beta-\alpha)<0, g g \rightarrow h^{0}$ is suppressed compared to the SM value due to cancellations between the $\cos (\beta-\alpha)$ and $\sin (\beta-\alpha)$ terms in the top Yukawa coupling, as shown in eq. (4.3). Note that we have shown the plots only for $\tan \beta \leq 4$ since the model is perturbatively valid only for $\tan \beta \lesssim 4$, as will be demonstrated below in the results of the full analysis. 

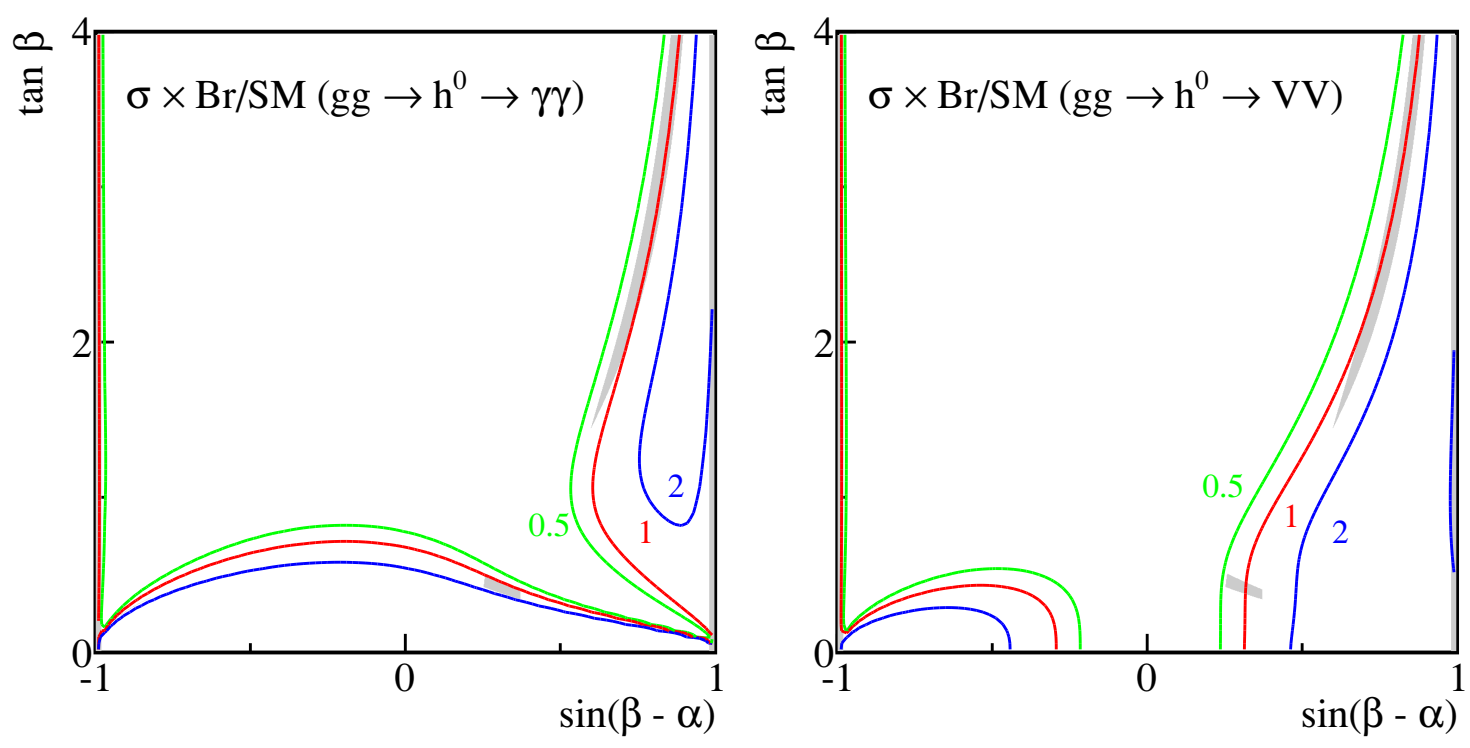

Figure 3. $\sigma \times \mathrm{Br} / \mathrm{SM}$ for the processes $g g \rightarrow h^{0} \rightarrow \gamma \gamma$ (left), and $g g \rightarrow h^{0} \rightarrow W W / Z Z$ (right) in the $h^{0}-126$ case. The contour lines are $\sigma \times \mathrm{Br} / \mathrm{SM}=0.5$ (green), 1 (red), and 2 (blue). The shaded gray are regions where cross sections of $\gamma \gamma$ and $W W / Z Z$ channels satisfy eq. (3.7).

The $h^{0}$ decay branching fractions $h^{0} \rightarrow V V, \gamma \gamma$ can be written approximately as

$$
\frac{\operatorname{Br}\left(h^{0} \rightarrow X X\right)}{\operatorname{Br}\left(h_{\mathrm{SM}} \rightarrow X X\right)}=\frac{\Gamma_{X X}}{\Gamma_{\text {total }}} \times \frac{\Gamma_{\text {total }}^{\mathrm{SM}}}{\Gamma_{X X}^{\mathrm{SM}}} \approx\left\{\begin{array}{c}
\frac{\sin ^{2}(\beta-\alpha)}{\sin ^{2}(\beta-\alpha) \operatorname{Br}\left(h_{\mathrm{SM}} \rightarrow V V\right)+\frac{\sin ^{2} \alpha}{\cos ^{2} \beta} \operatorname{Br}\left(h_{\mathrm{SM}} \rightarrow b b\right)+\ldots} \\
\frac{\Gamma\left(h^{0} \rightarrow \gamma \gamma\right) / \Gamma\left(h_{\mathrm{SM}} \rightarrow \gamma \gamma\right)}{\sin ^{2}(\beta-\alpha) \operatorname{Br}\left(h_{\mathrm{SM}} \rightarrow V V\right)+\frac{\sin ^{2} \alpha}{\cos ^{2} \beta} \operatorname{Br}\left(h_{\mathrm{SM}} \rightarrow b b\right)+\ldots}
\end{array},\right.
$$

where we have explicitly listed the dominant $b b$ and $W W / Z Z$ channels and used "+.." to indicate other sub-dominant SM Higgs decay channels.

In the right panel of figure 2, we show contours of $\mathrm{Br} / \mathrm{Br}_{\mathrm{SM}}$ for $V V$ (solid lines) and $\gamma \gamma$ (dashed lines) channels. Both $V V$ and loop induced (dominantly $W$-loop) $\gamma \gamma$ channels exhibit similar parameter dependence on $\tan \beta$ and $\sin (\beta-\alpha)$ since both channels are dominantly controlled by the same $h^{0} V V$ coupling. While contours of $\mathrm{Br} / \mathrm{Br}_{\mathrm{SM}} \gtrsim 1$ appear near $\sin (\beta-\alpha) \sim \pm 1$ for unsuppressed $h^{0} V V$ couplings, $h^{0} \rightarrow \gamma \gamma$ shows some spread for negative $\sin (\beta-\alpha)$ and small $\tan \beta$ due to the correction to top Yukawa in the loop-indued $h^{0} \gamma \gamma$ coupling.

Combining both the production and the decay branching fractions, we present the contours of $\sigma \times \mathrm{Br} / \mathrm{SM}$ in figure 3 for $\gamma \gamma$ (left panel) and $V V$ (right panel) for $\sigma \times \mathrm{Br} / \mathrm{SM}=$ 0.5 (green), 1 (red), and 2 (blue). Once we demand that the cross sections for these processes be consistent with the experimental observation of a $126 \mathrm{GeV}$ Higgs, as given in eq. (3.7), the allowed regions of parameter space split into four distinct regions, as indicated by the shaded gray areas. There are two narrow regions one each at $\sin (\beta-\alpha)= \pm 1$ (the gray regions at $\sin (\beta-\alpha)= \pm 1$ overlap with the picture frame boundary and are therefore hard to see), one extended region of $0.55<\sin (\beta-\alpha)<0.9$, and one low $\tan \beta$ region around $\sin (\beta-\alpha) \sim 0.3$ for $\tan \beta \sim 0.5$. Constraints from $R_{b}$ disfavor this low $\tan \beta$ region and 

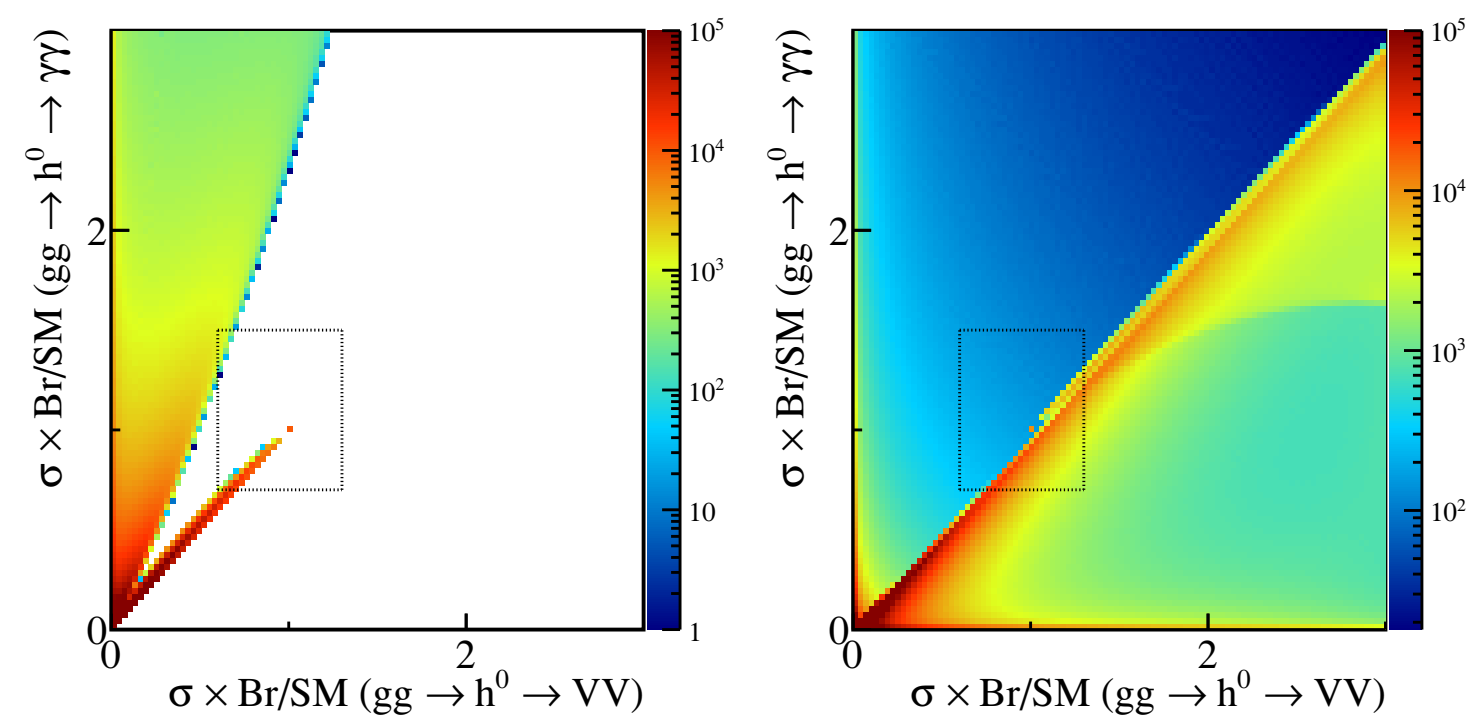

Figure 4. $\sigma \times \mathrm{Br} / \mathrm{SM}$ for $g g \rightarrow h^{0} \rightarrow \gamma \gamma$ versus $g g \rightarrow h^{0} \rightarrow V V$ for negative $\sin (\beta-\alpha)$ (left panel), and positive $\sin (\beta-\alpha)$ (right panel) in the $h^{0}-126$ case. Color map indicates the density of points with red being the most dense region and blue being the least dense region. Also indicated by the small rectangular box is the normalized signal cross section range of $\gamma \gamma$ between 0.7 and 1.5 , and $V V$ channels between 0.6 and $1.3[4,7]$.

therefore we will not discuss it further. In what follows, we will display separate plots for positive and negative $\sin (\beta-\alpha)$ to show the different features that appear in these two cases.

In figure 4, we show the correlations for $\sigma \times \mathrm{Br} / \mathrm{SM}$ for the $\gamma \gamma$ channel against $V V$, for negative (positive) values of $\sin (\beta-\alpha)$ in the left (right) panel as a density plot. Color coding is such that the points in red are the most dense (i.e., most likely) and points in blue are the least dense (i.e., less likely). Also indicated by the small rectangular box is the normalized signal cross section range of $\gamma \gamma$ between 0.7 and 1.5, and $V V$ channels between 0.6 and 1.3 , as given in eq. (3.7) $[4,7]$. Note that the corresponding signal windows in $\tan \beta$ versus $\sin (\beta-\alpha)$ plane are also sketched in figure 3 as the shaded gray regions. For negative $\sin (\beta-\alpha)$, there are two branches: the one along the diagonal line with $\gamma \gamma: V V \sim 1$ and $\sigma_{\gamma \gamma} \lesssim 1$, which can be mapped on to the $\sin (\beta-\alpha)=-1$ branch in figure 3 . The other branch in the upper-half plane where $\gamma \gamma: V V \gtrsim 2$ and $\sigma_{\gamma \gamma}$ extends to 2 or larger is strongly disfavored given the current observed Higgs signal region.

For positive values of $\sin (\beta-\alpha)$, the diagonal region is the most probable, with $\gamma \gamma: V V \lesssim 1$ and $\sigma_{\gamma \gamma}$ possibly extending over a relatively large range around 1 . Branches with $\sigma_{\gamma \gamma}$ or $\sigma_{V V} \sim 0$ along the axes are strongly disfavored given the current observation of the Higgs signal.

Thus we see that for all values of $\sin (\beta-\alpha)$, the $V V$ and $\gamma \gamma$ channels are positively correlated. ${ }^{4}$ Most of the points falls into $\gamma \gamma: V V \sim 1$ with the cross section of both around the SM strength. This means that an excess in the $\gamma \gamma$ channel should most likely

\footnotetext{
${ }^{4}$ This agrees with the results of $[15]$.
} 

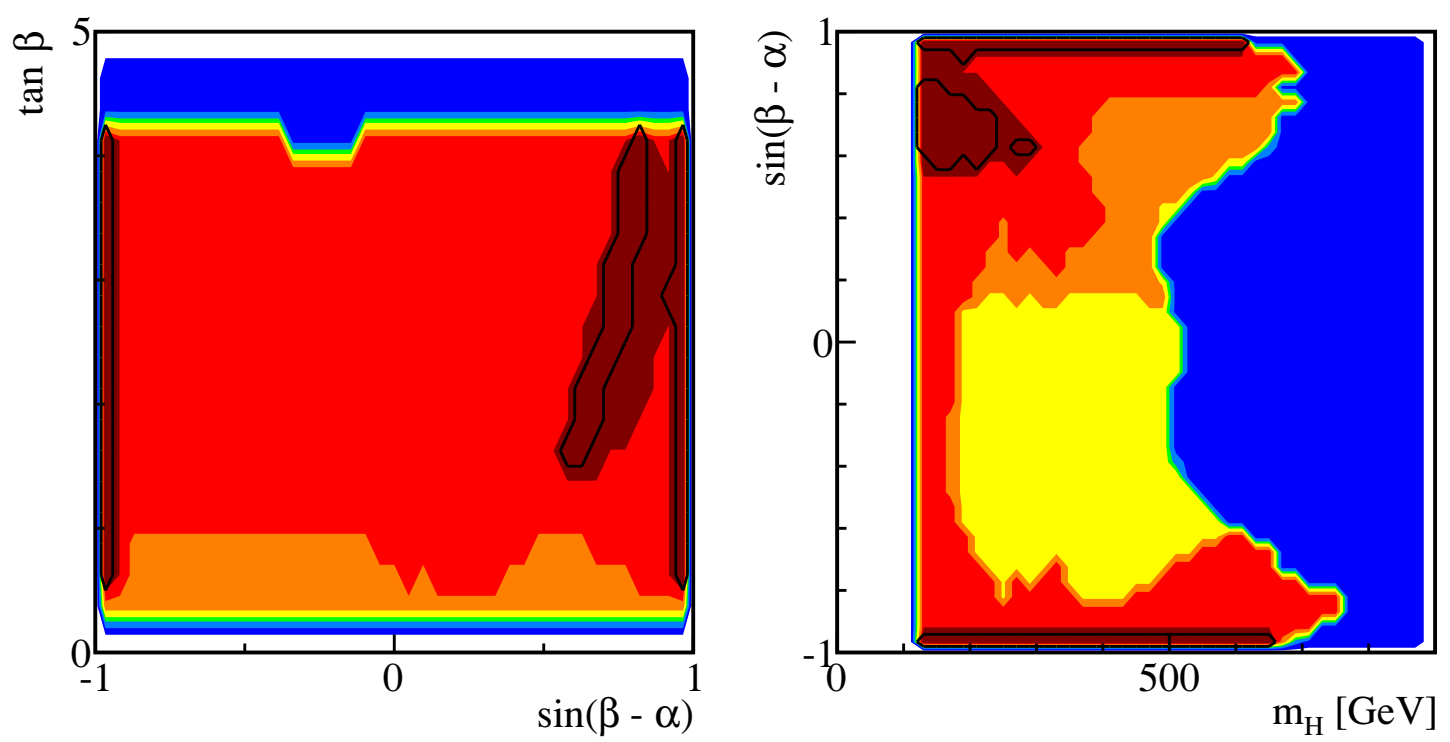

Figure 5. Parameter regions in the $h^{0}-126$ case for $\tan \beta$ versus $\sin (\beta-\alpha)$ (left panel) and $\sin (\beta-\alpha)$ versus $m_{H}$ (right panel). We show regions excluded by stability, unitarity and perturbativity (dark blue), $S$ and $T$ (light blue), LEP results (green), Tevatron and LHC results (yellow), and $R_{b}$ (orange). Regions that survive all the theoretical and experimental constraints are shown in red. Also shown in dark red are regions consistent with the light CP-even Higgs interpreted as the observed $126 \mathrm{GeV}$ scalar resonance, satisfying the cross section requirement of eq. (3.7) for $g g \rightarrow h^{0} \rightarrow$ $\gamma \gamma, W W / Z Z$. Regions enclosed by the black curves are the ones that survive the flavor constraints.

be accompanied by an excess in the $Z Z$ and $W W$ channels, and this fact serves as an important piece of discrimination for this model as more data is accumulated.

The above analysis illustrates the cross section and decay branching fraction behavior of the light CP-even Higgs when it is interpreted as the observed $126 \mathrm{GeV}$ SM-like Higgs, using the approximate formulae in eqs. (4.2)-(4.4). Note that we have only included the usual SM Higgs decay channels in $\Gamma_{\text {total }}$ in eq. (4.4). While it is a valid approximation in most regions of the parameter space, it might break down when light states in the spectrum open up new decay modes or introduce large loop contributions to either $g g \rightarrow h^{0}$ or $h^{0} \rightarrow \gamma \gamma$. In our full analysis presented below with scanning over the parameter spaces, we used the program 2HDMC, which takes into account all the decay channels of the Higgs, as well as other loop corrections to the gluon fusion production or Higgs decays to $\gamma \gamma$.

\subsection{Parameter spaces}

Fixing $m_{h}=126 \mathrm{GeV}$ still leaves us with five parameters: three masses, $m_{H}, m_{A}, m_{H^{ \pm}}$, and two angles $\tan \beta$ and $\sin (\beta-\alpha)$. Varying those parameters in the ranges given in eqs. (3.1)(3.4), we now study the remaining parameter regions satisfying all the theoretical and experimental constraints as well as regions that are consistent with the observed Higgs signal.

The left panel of figure 5 shows the viable regions in $\tan \beta$ versus $\sin (\beta-\alpha)$ plane when various theoretical constraints and experimental bounds are imposed sequentially. The red regions are those that satisfy all the constraints. Also shown in dark red are regions 

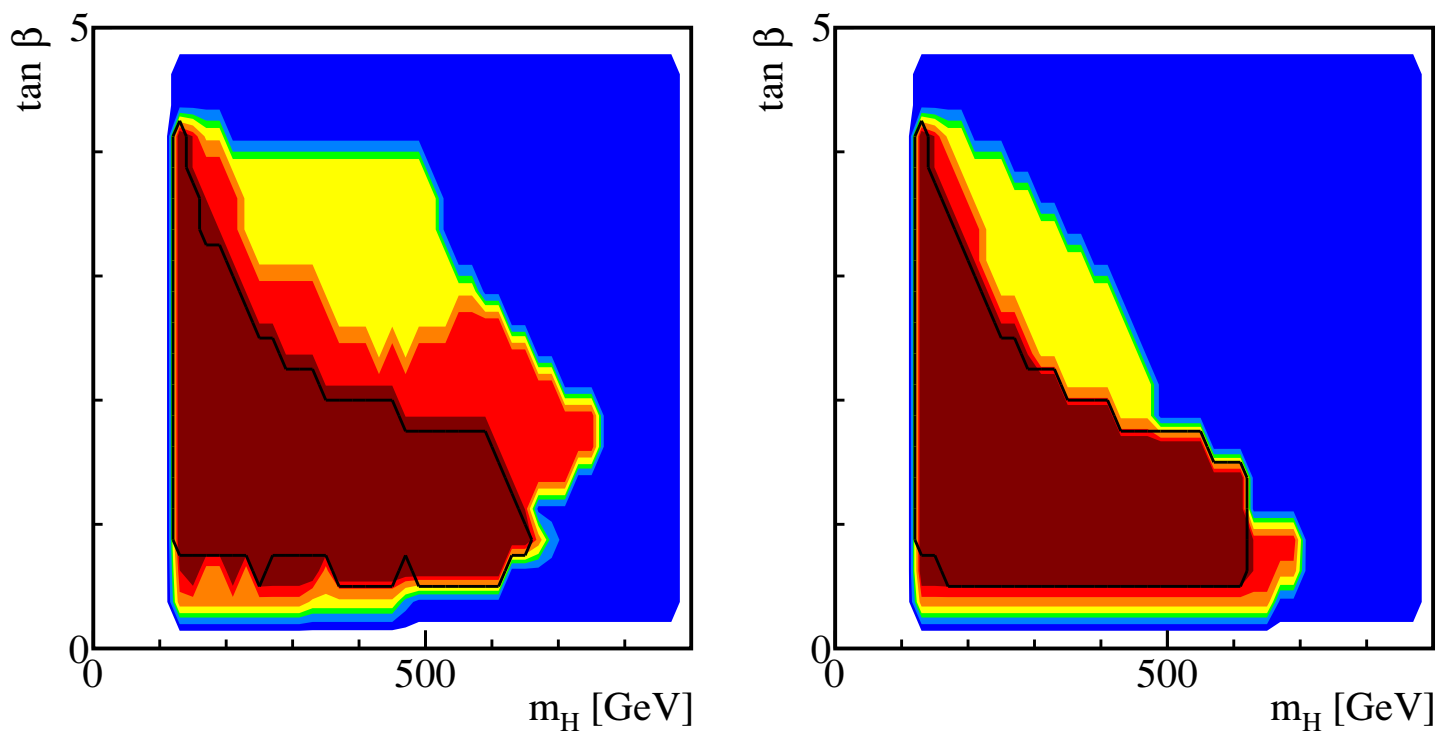

Figure 6. Parameter regions in the $h^{0}-126$ case for $\tan \beta$ versus $m_{H}$ with $\sin (\beta-\alpha)<0$ (left panel) and $\sin (\beta-\alpha)>0$ (right panel). Color coding is the same as figure 5 .

consistent with the light CP-even Higgs interpreted as the observed $126 \mathrm{GeV}$ scalar particle, satisfying the cross section requirement of eq. (3.7) for $g g \rightarrow h^{0} \rightarrow \gamma \gamma, W W / Z Z$. The signal regions (two narrow regions at $\sin (\beta-\alpha)= \pm 1$, and one extended region with $0.55<\sin (\beta-$ $\alpha)<0.9)$ agree well with the shaded region in figure 3 . The small region around $\sin (\beta-\alpha) \sim$ 0.3, however, disappeared, due to the $R_{b}$ constraint [68]. Regions with $\tan \beta \gtrsim 4$ are excluded by perturbative bounds since one of $\lambda_{1,2}$ becomes non-perturbative for larger value of $\tan \beta(\cos \beta \rightarrow 0)$, as shown in eq. (2.4). Consequently, the bottom loop contribution to the gluon fusion production cross section [8] is not a major factor for the $h^{0}-126$ case.

To further explore the flavor constraints, we show in figure 5 the regions enclosed by the black curves being those that survive the flavor bounds. As can clearly be seen, flavor bounds do not significantly impact the surviving signal regions.

The right panel of figure 5 shows the allowed region in the $\sin (\beta-\alpha)-m_{H}$ plane. Imposing all the theoretical constraints, in particular, the perturbativity requirement, translates into an upper bound on $m_{H}$ of around $750 \mathrm{GeV}$. Higgs search bounds from the LHC removes a large region in negative $\sin (\beta-\alpha)$, mostly from the stringent bounds from $W W$ and $Z Z$ channels for the heavy Higgs. The positive $\sin (\beta-\alpha)$ region is less constrained since $g g \rightarrow H^{0} \rightarrow W W / Z Z$ are much more suppressed. $R_{b}$, in addition, excludes part of the positive $\sin (\beta-\alpha)$ region with relatively large $m_{H}$. Requiring $h^{0}$ to fit the observed Higgs signal further narrows down the favored regions, as shown in dark red. For $\sin (\beta-\alpha)= \pm 1$, $m_{H}$ could be as large as $650 \mathrm{GeV}$. For $0.55 \lesssim \sin (\beta-\alpha) \lesssim 0.9, m_{H}$ is constrained to be less than $300 \mathrm{GeV}$. The correlation between $m_{H}$ and $\sin (\beta-\alpha)$ indicates that if a heavy CP-even Higgs is discovered to be between 300 and $650 \mathrm{GeV}, \sin (\beta-\alpha)$ is constrained to be very close to \pm 1 , indicating the light Higgs has SM-like couplings to the gauge sector. 

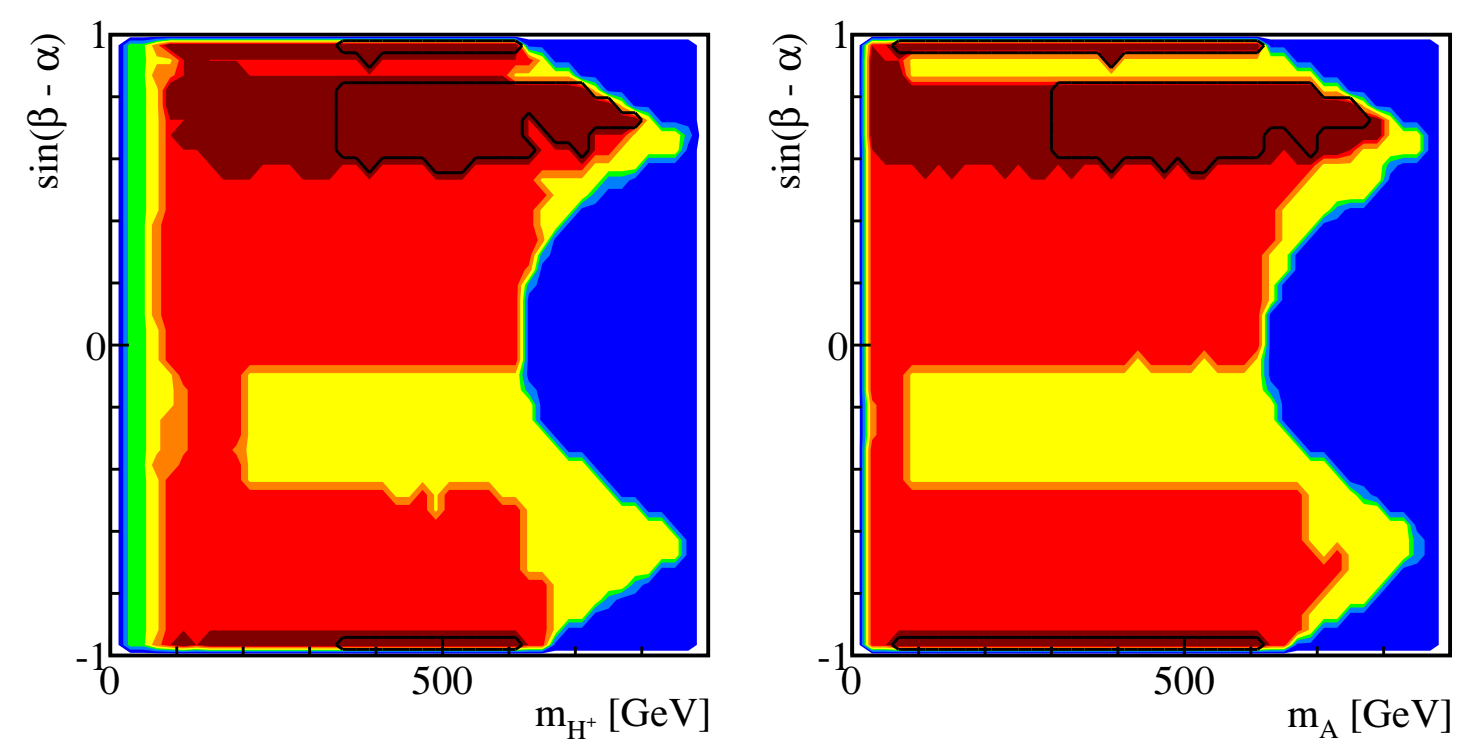

Figure 7. Parameter regions in the $h^{0}-126$ case for $\sin (\beta-\alpha)$ versus $m_{H^{ \pm}}$(left panel) and $m_{A}$ (right panel). Color coding is the same as figure 5 .

In figure 6 , we present the parameter regions for $\tan \beta$ versus $m_{H}$ with $\sin (\beta-\alpha)<0$ (left panel) and $\sin (\beta-\alpha)>0$ (right panel). Regions with large $m_{H}$ are typically realized for small $\tan \beta$ roughly between 1 and 2 . There are also noticeable difference for positive or negative $\sin (\beta-\alpha)$ for regions that survive all the experimental constraints (red regions). Negative $\sin (\beta-\alpha)$ allows larger values of $\tan \beta$ for a given mass of $m_{H}$. Small values of $\tan \beta$ is disfavored by the perturbativity of top Yukawa coupling [39], $R_{b}$ [68], and the flavor constraints [78].

Figure 7 shows the parameter regions in $\sin (\beta-\alpha)$ versus $m_{H^{ \pm}}$(left panel) and $m_{A}$ (right panel). For negative $\sin (\beta-\alpha)$ between -0.5 to -0.1 , only regions with $m_{A}<60 \mathrm{GeV}$ survive the LHC Higgs search bounds. This is because $H^{0} \rightarrow A^{0} A^{0}$ opens up in this region, which leads to the suppression of $H^{0} \rightarrow W W / Z Z$ allowing it to escape the experimental constraints. The corresponding surviving region in $120 \mathrm{GeV}<m_{H^{ \pm}}<200 \mathrm{GeV}$ is introduced by the correlation between $m_{A}$ and $m_{H^{ \pm}}$due to $\Delta \rho$ constraints. Imposing the cross section requirement for $h^{0}$ to satisfy the Higgs signal region results in three bands in both $m_{A}$ and $m_{H^{ \pm}}$, with masses extending all the way to about $800 \mathrm{GeV}$. Imposing the flavor constraints leaves regions with $m_{H^{ \pm}} \gtrsim 300 \mathrm{GeV}$ viable for $\sin (\beta-\alpha)= \pm 1$ or $\sin (\beta-\alpha)$ between 0.55 and 0.9 , while even smaller values for $m_{A}$ remain viable at $\sin (\beta-\alpha)= \pm 1$.

The allowed regions in the $\tan \beta-m_{H^{ \pm}}$and $\tan \beta-m_{A}$ planes share similar features before flavor constraints are taken into account, which are shown in figure 8 . The top two panels show the allowed regions in the $\tan \beta-m_{H^{ \pm}}$plane for negative and positive $\sin (\beta-\alpha)$, while the lower two panels are for $\tan \beta-m_{A}$. LEP places a lower bound on the charged Higgs mass around $80 \mathrm{GeV}[55,56]$. In the signal region for $\sin (\beta-\alpha)<0$, both $m_{H^{ \pm}}$and $m_{A}$ are less than about $600 \mathrm{GeV}$, while their masses could be extended to $800 \mathrm{GeV}$ for $\sin (\beta-\alpha)>0$ and $\tan \beta>2$. The difference between the $m_{A}$ range for different 

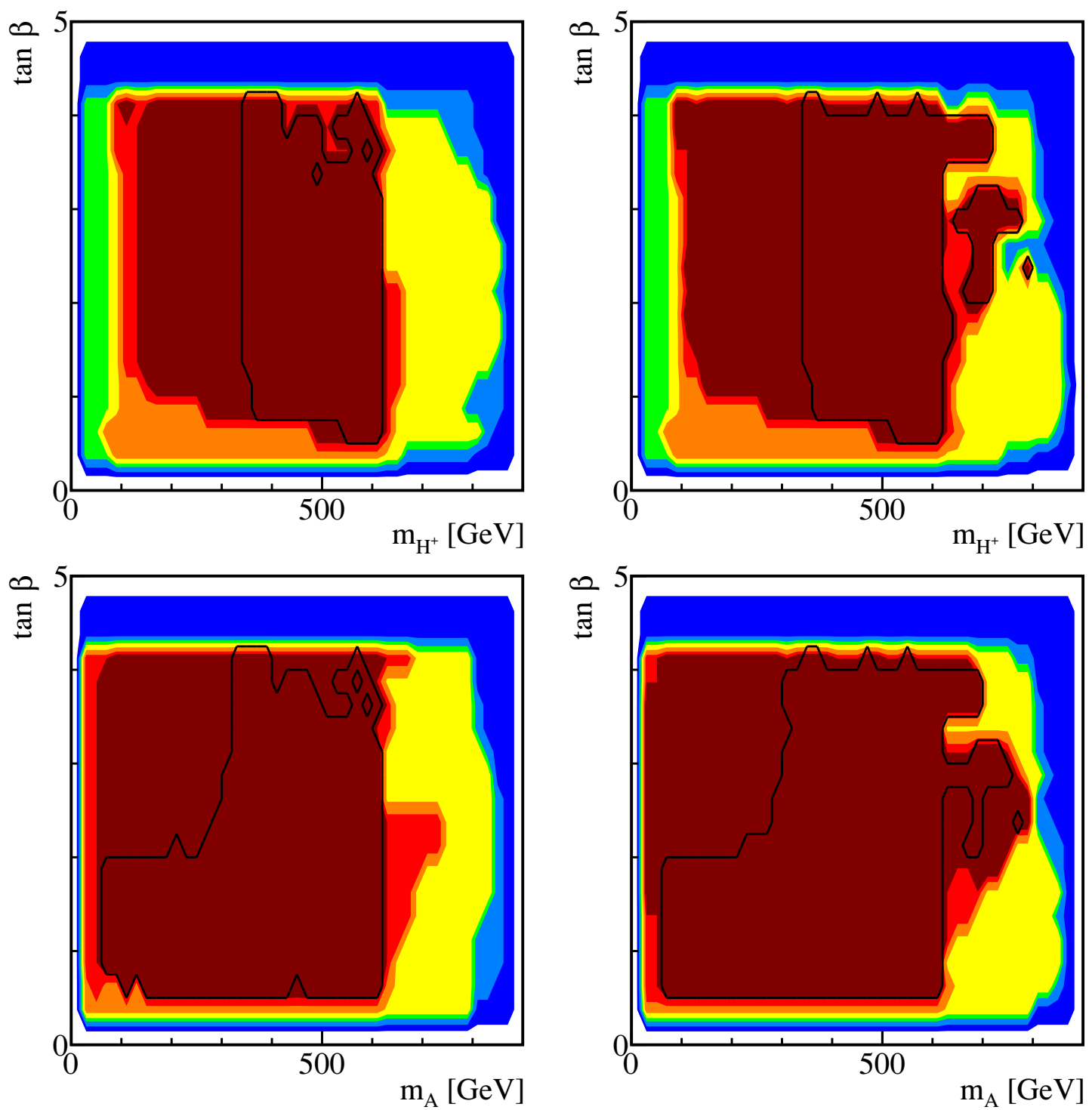

Figure 8. Parameter regions in the $h^{0}-126$ case for $\tan \beta$ versus $m_{H^{ \pm}}$(top panels) and $m_{A}$ (lower panels) with $\sin (\beta-\alpha)<0$ (left panels) and $\sin (\beta-\alpha)>0$ (right panels). Color coding is the same as figure 5 .

signs of $\sin (\beta-\alpha)$ can be explained as follows: regions with $m_{A}>600 \mathrm{GeV}$ can only occur for $|\sin (\beta-\alpha)|$ between 0.4 and 0.8 , as shown in the right panel of figure 7 . The Higgs signal region of $\tan \beta$ versus $\sin (\beta-\alpha)$ (left panel of figure 5 ) shows that to simultaneously satisfy both the $\tan \beta$ range and $\sin (\beta-\alpha)$ range, only positive $\sin (\beta-\alpha)$ case survives.

Flavor bounds, as expected, have a marked effect here ruling out any value of $m_{H^{ \pm}} \lesssim$ $300 \mathrm{GeV}$ for all values of $\tan \beta$, mainly due to the $b \rightarrow s \gamma$ constraint. For the CP-odd Higgs, only a corner of $\tan \beta>2$ and $m_{A}<300 \mathrm{GeV}$ is excluded, due to the combination of flavor and $\Delta \rho$ constraints. As shown in figure 6 , only relatively light $m_{H} \lesssim 300 \mathrm{GeV}$ is allowed for $\tan \beta>2$. The flavor constraints of $m_{H^{ \pm}} \gtrsim 300 \mathrm{GeV}$ is then translated to $m_{A} \gtrsim 300 \mathrm{GeV}$ 

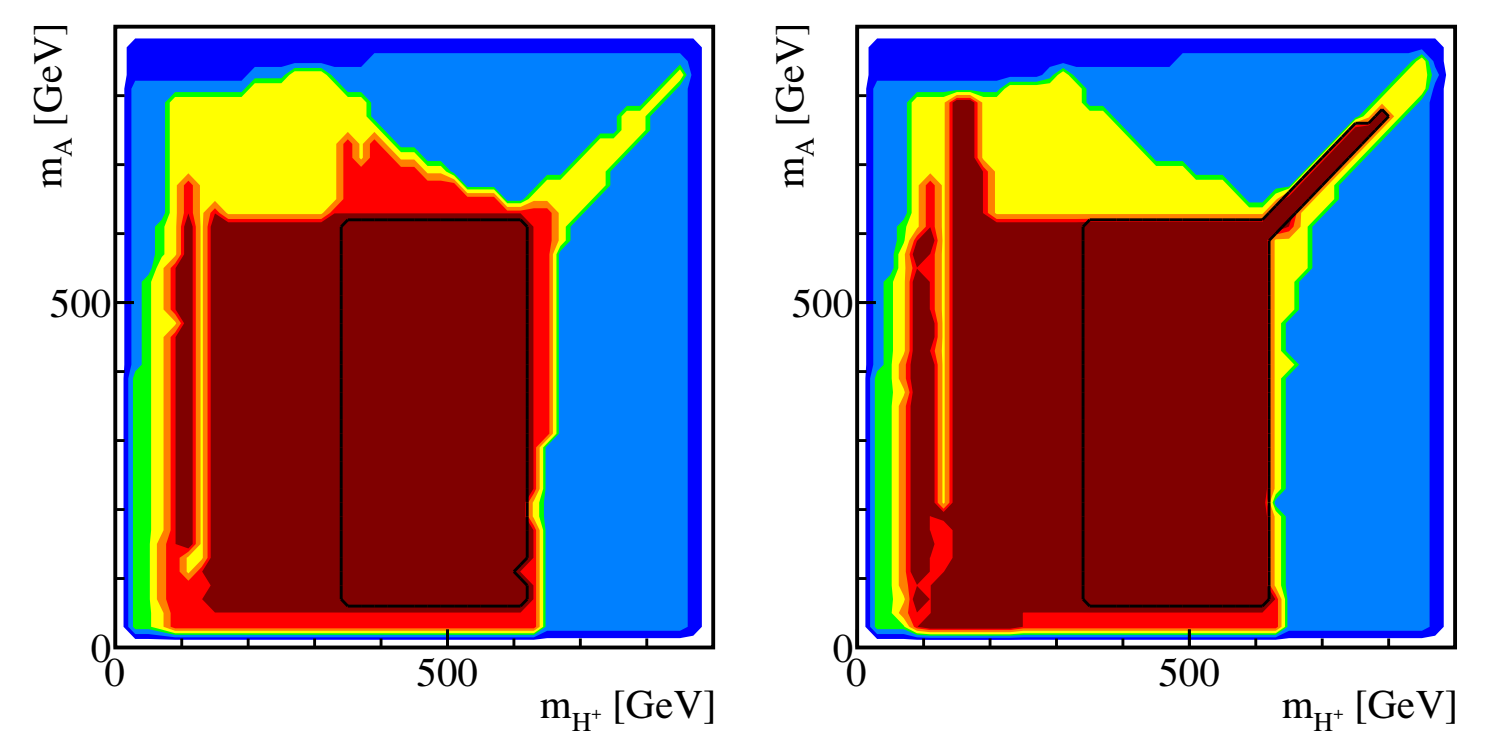

Figure 9. Parameter regions in the $h^{0}-126$ case for $m_{A}$ versus $m_{H^{ \pm}}$with $\sin (\beta-\alpha)<0$ (left panel) and $\sin (\beta-\alpha)>0$ (right panel). Color coding is the same as figure 5 .

since the difference between $m_{A}$ and $m_{H^{ \pm}}$is constrained by $\Delta \rho$ considerations when both $m_{h}$ and $m_{H}$ are relatively small. For $\tan \beta<2, m_{H}$ could be relatively high, which cancels the large contribution to $\Delta \rho$ from large $m_{H^{ \pm}}$while allowing $m_{A}$ to be light.

In figure 9 , we present the parameter regions in the $m_{A}-m_{H^{ \pm}}$plane for negative and positive values of $\sin (\beta-\alpha) \cdot m_{A}$ and $m_{H^{ \pm}}$are uncorrelated for most parts of the parameter space. For $\sin (\beta-\alpha)>0$ when $m_{A, H^{ \pm}}$could reach values larger than $600 \mathrm{GeV}, \tan \beta$ is at least 2 or larger (see figure 8). $m_{H}$ is restricted to less than $300 \mathrm{GeV}$ in this region, which results in a strong correlation between $m_{A}$ and $m_{H^{ \pm}}$due to the $\Delta \rho$ constraints.

Figure 10 shows the parameter space in the $m_{A}-m_{H}$ plane for negative (left panel) and positive (right panel) $\sin (\beta-\alpha)$. These two masses are largely uncorrelated for either sign of $\sin (\beta-\alpha)$. Note that for $\sin (\beta-\alpha)>0$, large $m_{A}$ between $600-800 \mathrm{GeV}$ is only possible for small values of $m_{H} \lesssim 250 \mathrm{GeV}$. This is because the corresponding $\tan \beta$ is larger than 2 , which bounds $m_{H}$ from above. The lower-left corners excluded by flavor constraints correspond to the upper-left corners in $m_{A}-\tan \beta$ plots in figure 8 , since at least one of $m_{A}$ or $m_{H}$ would need to be relatively heavy to cancel the contribution to $\Delta \rho$ from $m_{H^{ \pm}}>300 \mathrm{GeV}$.

We conclude this section with the following comments:

- If $h^{0}$ is the $126 \mathrm{GeV}$ resonance, then the $\gamma \gamma$ channel is closely correlated with $W W / Z Z$. Specifically, a moderate excess in $\gamma \gamma$ should be accompanied by a corresponding excess in $W W / Z Z$.

- The combination of all theoretical constraints requires $\tan \beta \lesssim 4$. Therefore, the bottom-loop enhancement to the gluon fusion [8] is never a major factor. Regions of $\sin (\beta-\alpha)$ and $\tan \beta$ are highly restricted once we require the light CP-even Higgs to be the observed $126 \mathrm{GeV}$ scalar particle: $\tan \beta$ between 0.5 to 4 for $\sin (\beta-\alpha)= \pm 1$, $\tan \beta$ between 1.5 to 4 for $0.55<\sin (\beta-\alpha)<0.9$. The masses of the other Higgses, 

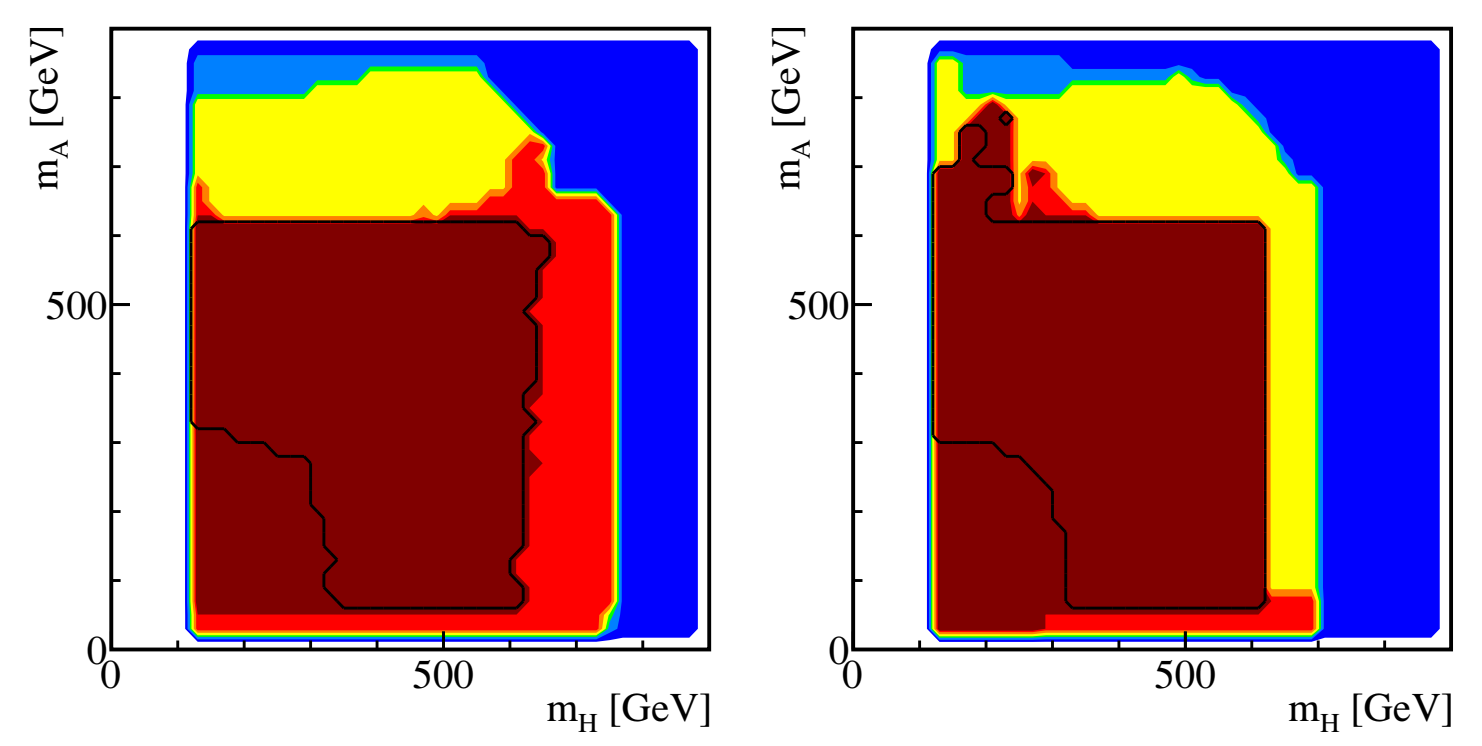

Figure 10. Parameter regions in the $h^{0}-126$ case for $m_{A}$ versus $m_{H}$ with $\sin (\beta-\alpha)<0$ (left panel) and $\sin (\beta-\alpha)>0$ (right panel). Color coding is the same as figure 5 .

$m_{H}, m_{A}$, and $m_{H^{ \pm}}$, however, are largely unrestricted and uncorrelated, except for the region where $\sin (\beta-\alpha)>0$ and $m_{A, H^{ \pm}} \gtrsim 600 \mathrm{GeV}$, which exhibits a strong correlation between these two masses.

- The discovery of any one of the extra scalars can largely narrow down the parameter space, in particular, if the masses of those particles are relatively high.

- Flavor bounds do not change the allowed parameter space much except for the charged Higgs mass, which is constrained to lie above $300 \mathrm{GeV}$.

\section{Heavy Higgs at $126 \mathrm{GeV}$}

\subsection{Cross sections and correlations}

It is possible that the $126 \mathrm{GeV}$ resonance discovered at the LHC corresponds to the heavier of the two CP-even Higgses, $H^{0}$. There are a few noticeable changes for the heavy $H^{0}$ being the SM-like Higgs boson. First of all, since the coupling of the heavy Higgs to a gauge boson pair is scaled by a factor of $\cos (\beta-\alpha)$ as opposed to $\sin (\beta-\alpha)$, demanding SM-like cross sections for $H^{0}$ forces us to consider $\sin (\beta-\alpha) \sim 0$, as opposed to $\sin (\beta-\alpha) \sim \pm 1$ in the $h^{0}-126$ case. Secondly, as will be demonstrated below, the bottom contribution to the gluon fusion production could be significantly enhanced since the range of $\tan \beta$ could be much larger compared to the $h^{0}-126$ case.

Similar to eqs. (4.2) and (4.3) in section 4, the ratios of the gluon fusion cross sections normalized to the SM can be written approximately as:

$$
\frac{\sigma\left(g g \rightarrow H^{0}\right)}{\sigma_{\mathrm{SM}}}=\frac{\sin ^{2} \alpha}{\sin ^{2} \beta}+\frac{\cos ^{2} \alpha}{\cos ^{2} \beta} \frac{\left|A_{1 / 2}\left(\tau_{b}\right)\right|^{2}}{\left|A_{1 / 2}\left(\tau_{t}\right)\right|^{2}}
$$



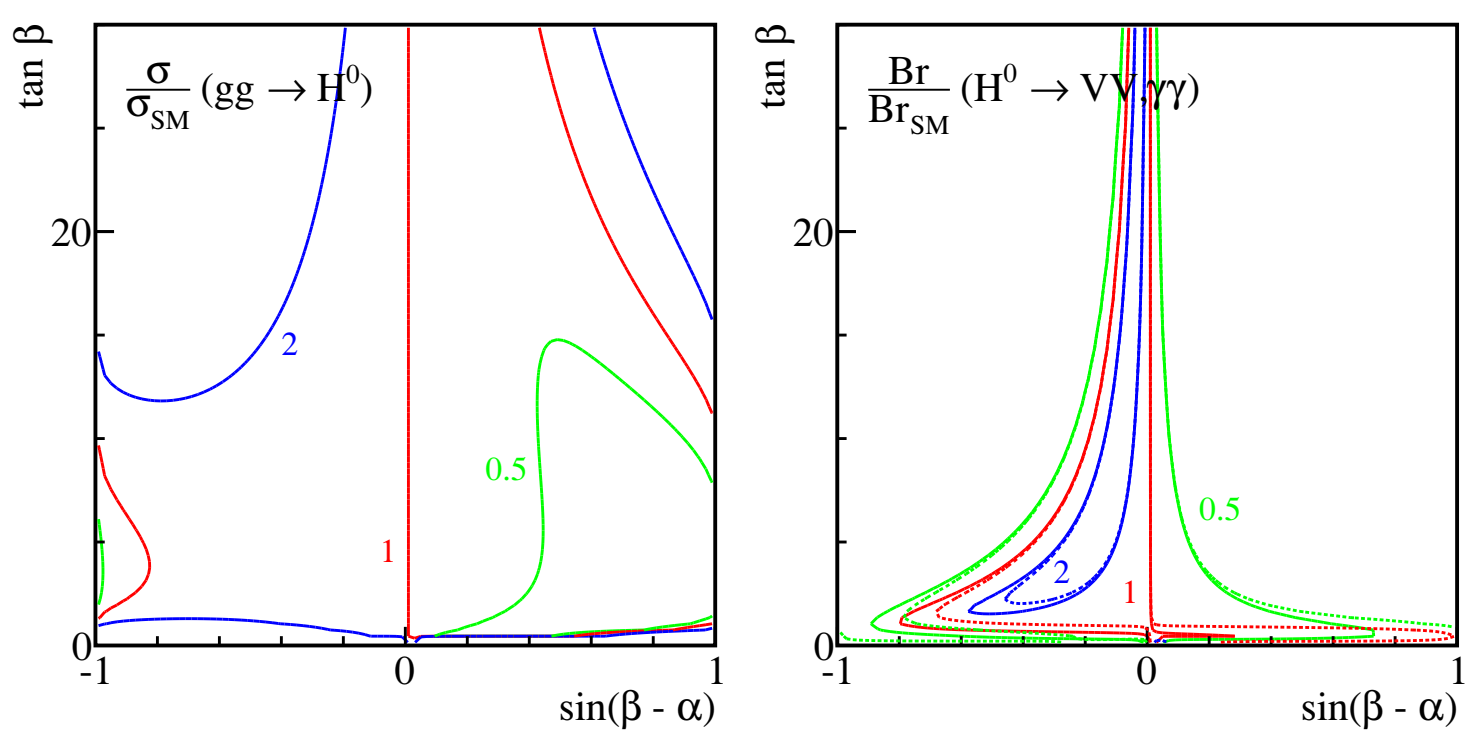

Figure 11. The normalized $g g \rightarrow H^{0}$ production cross section contours (left panel) and $H^{0} \rightarrow V V$ (solid lines of the right panel) and $H^{0} \rightarrow \gamma \gamma$ (dashed lines of the right panel) branching fractions in the $H^{0}-126$ case. The contour lines are $\sigma / \sigma_{\mathrm{SM}}, \mathrm{Br} / \mathrm{Br}_{\mathrm{SM}}=0.5$ (green), 1 (red), and 2 (blue).

$$
=\left[\frac{\sin (\beta-\alpha)}{\tan \beta}-\cos (\beta-\alpha)\right]^{2}+[\sin (\beta-\alpha) \tan \beta+\cos (\beta-\alpha)]^{2} \frac{\left|A_{1 / 2}\left(\tau_{b}\right)\right|^{2}}{\left|A_{1 / 2}\left(\tau_{t}\right)\right|^{2}} .
$$

Contours of $\sigma / \sigma_{\mathrm{SM}}\left(g g \rightarrow H^{0}\right)=0.5$ (green), 1 (red), and 2 (blue) are shown in the left panel of figure 11. $H^{0}$ couples exactly like the SM Higgs for $\sin (\beta-\alpha)=0$, while deviations from the $\mathrm{SM}$ values occur for $\sin (\beta-\alpha)$ away from zero. For $\sin (\beta-\alpha)<0, \sigma / \sigma_{\mathrm{SM}}\left(g g \rightarrow H^{0}\right)$ is almost always larger than 1 (except for a small region around $\sin (\beta-\alpha) \sim-1$ and $\tan \beta \lesssim 10$ ) while a suppression of the gluon fusion production is possible for positive values of $\sin (\beta-\alpha)$. This is due to cancellations between the $\sin (\beta-\alpha)$ and $\cos (\beta-\alpha)$ terms in the top Yukawa coupling, in particular, for low $\tan \beta$. The bottom loop contributes significantly when $\tan \beta$ is large, which enhances the gluon fusion production cross section.

$\operatorname{Br}\left(H^{0} \rightarrow V V, \gamma \gamma\right) / \mathrm{Br}_{\mathrm{SM}}$ can also be expressed similar to eq. (4.4):

$$
\frac{\mathrm{BR}\left(H^{0} \rightarrow X X\right)}{\operatorname{BR}\left(h_{\mathrm{SM}} \rightarrow X X\right)}=\frac{\Gamma_{X X}}{\Gamma_{\text {total }}} \times \frac{\Gamma_{\text {total }}^{\mathrm{SM}}}{\Gamma_{X X}^{\mathrm{SM}}}=\left\{\begin{array}{c}
\frac{\cos ^{2}(\beta-\alpha)}{\cos ^{2}(\beta-\alpha) \operatorname{Br}\left(h_{\mathrm{SM}} \rightarrow V V\right)+\frac{\cos ^{2} \alpha}{\cos ^{2} \beta} \operatorname{Br}\left(h_{\mathrm{SM}} \rightarrow b b\right)+\ldots} \\
\frac{\Gamma\left(H^{0} \rightarrow \gamma \gamma\right) / \Gamma\left(h_{\mathrm{SM}} \rightarrow \gamma \gamma\right)}{\cos ^{2}(\beta-\alpha) \operatorname{Br}\left(h_{\mathrm{SM}} \rightarrow V V\right)+\frac{\cos ^{2} \alpha}{\cos ^{2} \beta} \operatorname{Br}\left(h_{\mathrm{SM}} \rightarrow b b\right)+\ldots}
\end{array}\right.
$$

with the contour lines given in the right panel of figure 11. A relative enhancement of the branching fractions over the SM values are observed in extended region of negative $\sin (\beta-\alpha)$, while it is mostly suppressed for positive $\sin (\beta-\alpha)$.

Combining the production cross sections and the decay branching fractions, contours of $g g \rightarrow H^{0} \rightarrow X X$ are given in figure 12 for $\gamma \gamma$ (left panel) and $W W / Z Z$ channels (right panel). Requiring the cross section to be consistent with the observed Higgs signal: $0.7-$ 1.5 for the $\gamma \gamma$ channel and $0.6-1.3$ for the $W W / Z Z$ channel, results in two distinct regions: a region close to $\sin (\beta-\alpha) \sim 0$, and an extended region of $-0.8 \lesssim \sin (\beta-\alpha) \lesssim-0.05$. 

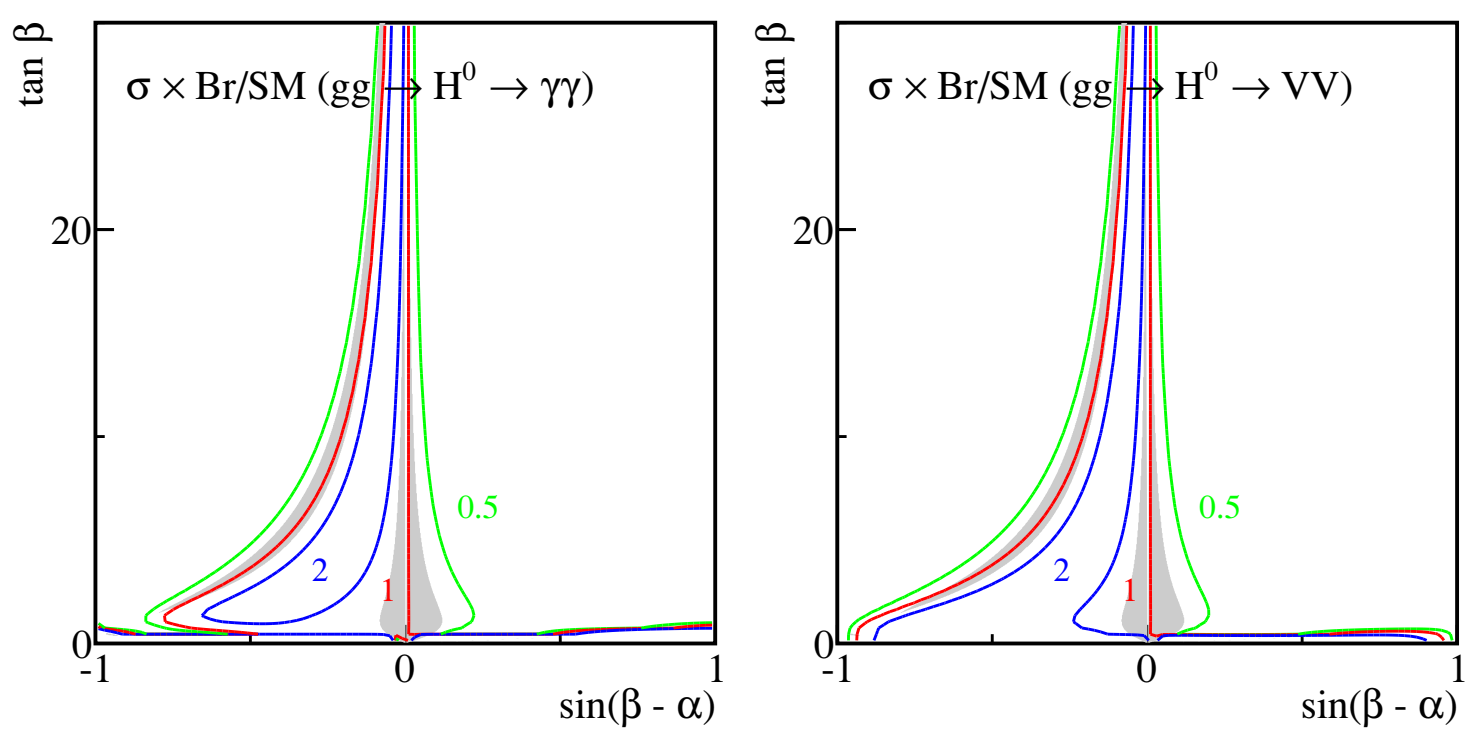

Figure 12. $\sigma \times \mathrm{Br} / \mathrm{SM}$ for the processes $g g \rightarrow H^{0} \rightarrow \gamma \gamma$ (left), and $g g \rightarrow H^{0} \rightarrow W W / Z Z$ (right) in the $H^{0}-126$ case. The contour lines are $\sigma \times \mathrm{Br} / \mathrm{SM}=0.5$ (green), 1 (red), and 2 (blue). The regions where cross sections of $\gamma \gamma$ and $W W / Z Z$ channels satisfy eq. (3.7) are shaded gray.

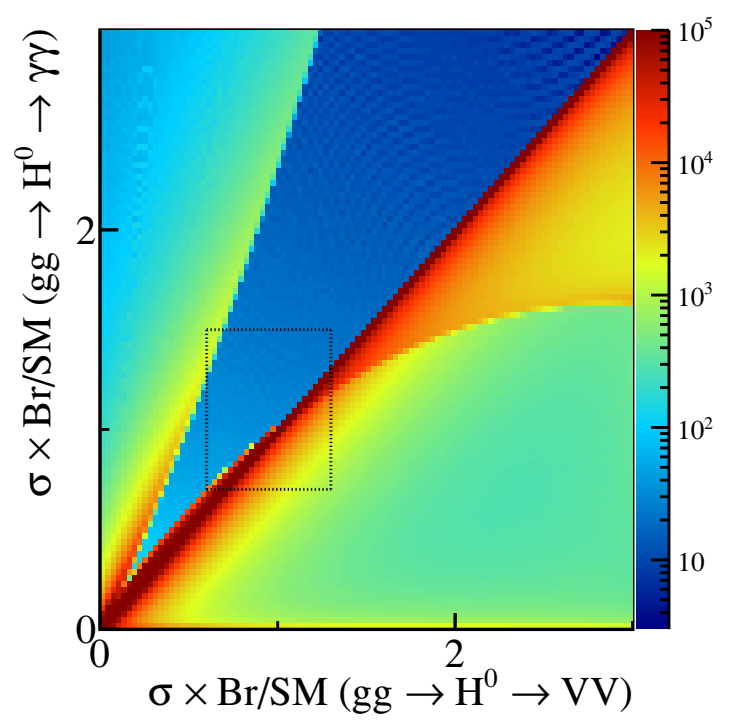

Figure 13. $\sigma \times \mathrm{Br} / \mathrm{SM}$ for $g g \rightarrow H^{0} \rightarrow \gamma \gamma$ versus $g g \rightarrow H^{0} \rightarrow V V$ in the $H^{0}-126$ case. Color coding is the same as in figure 4 . Also indicated by the small rectangular box is the normalized signal cross section range of $\gamma \gamma$ between 0.7 and 1.5, and $V V$ channels between 0.6 and $1.3[4,7]$.

Figure 13 shows the correlation between the $\gamma \gamma$ and $V V$ channels. Most of the points lie along the diagonal: $\gamma \gamma: V V \sim 1$. A second branch of $\gamma \gamma: W W \sim 2$ also appears, which corresponds to the very low $\tan \beta<1$ region in figure 12. This region is strongly constrained by $R_{b}$ and flavor bounds, and is therefore not considered further in our study. 


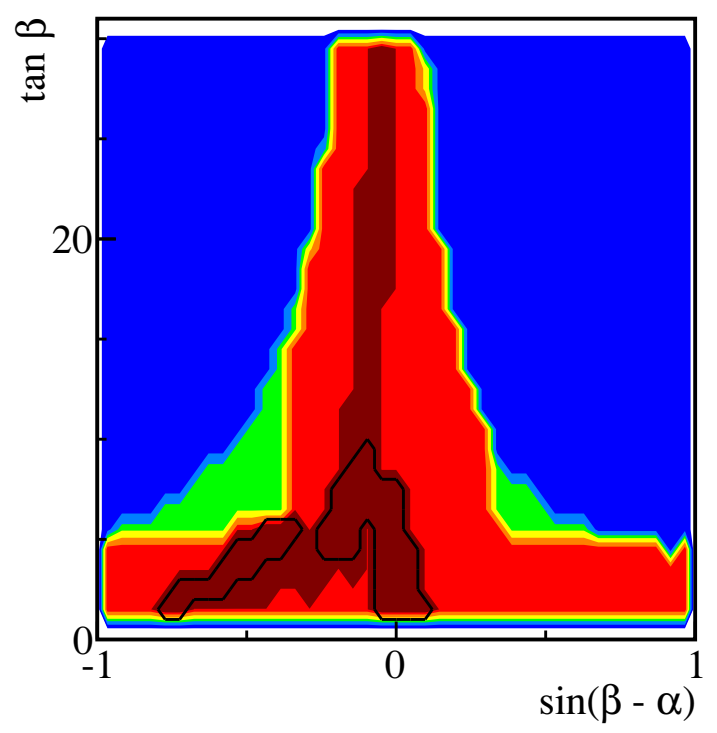

Figure 14. Parameter regions in the $H^{0}-126$ case for $\tan \beta$ versus $\sin (\beta-\alpha)$. Color coding is the same as figure 5 except that the dark red regions are the ones consistent with the heavy CP-even Higgs interpreted as the observed Higgs signal.

\subsection{Parameter spaces}

We now present the results for $H^{0}-126$ case with the full parameter scan, including all the theoretical and experimental constraints. Figure 14 presents the parameter regions in $\tan \beta$ versus $\sin (\beta-\alpha)$. The color coding is the same as in figure 5 , except that the signal regions in dark red are those with the heavy CP-even Higgs $H^{0}$ interpreted as the observed $126 \mathrm{GeV}$ scalar.

Requiring the heavy CP-even Higgs to satisfy the cross section ranges of the observed Higgs signal results in two signal regions: one region near $\sin (\beta-\alpha) \sim 0$ and an extended region of $-0.8 \lesssim \sin (\beta-\alpha) \lesssim-0.05$, consistent with figure 12 . Note however that the region around $\sin (\beta-\alpha) \sim 0$ is actually reduced to $\tan \beta \lesssim 8$. This is because larger values of $\tan \beta$ leads to smaller $m_{h}$ such that $m_{h}<m_{H} / 2$ (see right panel of figure 15 below). The opening of $H^{0} \rightarrow h^{0} h^{0}$ channel reduces the the branching fractions of $H^{0} \rightarrow W W / Z Z, \gamma \gamma$ forcing it outside the signal cross section region. Regions surviving the flavor bounds are the ones enclosed by black curves. Larger values of $\tan \beta \gtrsim 10$ are disfavored.

Figure 15 shows the parameter region in $\sin (\beta-\alpha)$ versus $m_{h}$ (left panel) and $\tan \beta$ versus $m_{h}$ (right panel). Within the narrow region around $\sin (\beta-\alpha) \sim 0, m_{h}$ can take all values up to $126 \mathrm{GeV}$. For $-0.8 \lesssim \sin (\beta-\alpha) \lesssim-0.35$, when the $H^{0} W W, H^{0} Z Z$ couplings could significantly deviate from the SM value while $h^{0} W W, h^{0} Z Z$ couplings are sizable, the light CP-even Higgs mass is constrained to be larger than about $80 \mathrm{GeV}$ from LEP Higgs searches [53, 54]. This is the interesting region where the two Higgses are close to being degenerate, with both $h^{0}$ and $H^{0}$ showing significant deviation of their couplings to gauge bosons from the SM value. 

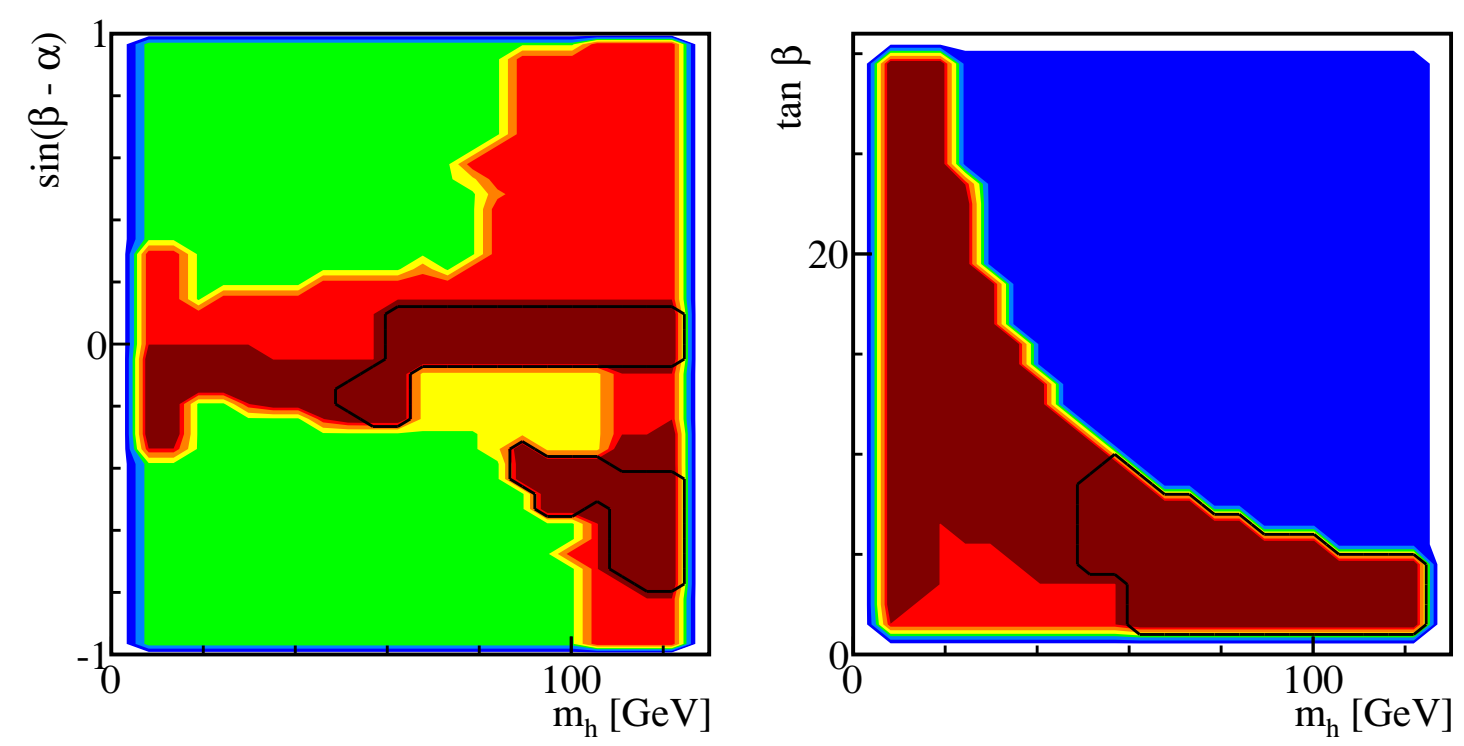

Figure 15. Parameter regions in the $H^{0}-126$ case for $\sin (\beta-\alpha)$ versus $m_{h}$ (left panel) and $\tan \beta$ versus $m_{h}$ (right panel). Color coding is the same as figure 14 .

The right panel of figure 15 shows the parameter region of $\tan \beta$ versus $m_{h}$. Larger values of $\tan \beta$ is only allowed for small values of $m_{h}$. The red region where $m_{h}<60 \mathrm{GeV}$ and $\tan \beta \lesssim 5$ can not satisfy the Higgs signal cross section requirement due to the opening of $H^{0} \rightarrow h^{0} h^{0}$ mode, which corresponds to the $m_{h}<60 \mathrm{GeV}, \sin (\beta-\alpha) \sim 0$ red region in the $\sin (\beta-\alpha)$ versus $m_{h}$ plot (left panel of figure 15). Imposing the flavor bounds further rules out regions with light $m_{h}$ below about $50 \mathrm{GeV}$, mainly due to the process $B_{s} \rightarrow \mu^{+} \mu^{-}$, as shown in the right panel of figure 1 . Large values of $\tan \beta \gtrsim 10$ are excluded correspondingly.

Figure 16 shows $\sin (\beta-\alpha)$ versus $m_{A, H^{ \pm}}$(left panels) and $\tan \beta$ versus $m_{A, H^{ \pm}}$(right panels). The plots for $m_{A}$ and $m_{H^{ \pm}}$are very similar, except for very low masses. Very large values of $m_{A, H^{ \pm}} \gtrsim 800 \mathrm{GeV}$ are excluded by theoretical considerations, similar to the $h^{0}-126$ case. $m_{A} \lesssim 60 \mathrm{GeV}$ and $\tan \beta \gtrsim 5$ are excluded by the LEP Higgs search [53], while the triangle region of $130 \lesssim m_{A} \lesssim 400 \mathrm{GeV}$ and $\tan \beta \gtrsim 13$ is excluded by the LHC searches for the CP-odd Higgs in $\tau \tau$ mode [58-64]. For the charged Higgs, small values of $m_{H^{ \pm}} \lesssim 80 \mathrm{GeV}$ are ruled out by LEP searches on charged Higgs [55, 56]. Tevatron and the LHC charged Higgs searches [58-64]: $t \rightarrow H^{ \pm} b \rightarrow \tau \nu_{\tau} b$ further rule out regions of $m_{H^{ \pm}} \lesssim 150 \mathrm{GeV}$ and $\tan \beta \gtrsim 17$. The triangle in $m_{H^{ \pm}}$versus $\tan \beta$ plot for $150 \mathrm{GeV}$ $\lesssim m_{H^{ \pm}} \lesssim 400 \mathrm{GeV}$ and $\tan \beta \gtrsim 13$ is translated from the corresponding region in $\tan \beta$ versus $m_{A}$, due to the correlation between $m_{A}$ and $m_{H^{ \pm}}$introduced by $\Delta \rho$, as shown below in figure 17. Imposing the flavor constraints further limits $m_{A} \gtrsim 300 \mathrm{GeV}, m_{H^{ \pm}} \gtrsim 300 \mathrm{GeV}$ and $\tan \beta \lesssim 10$.

$m_{A}$ and $m_{H^{ \pm}}$exhibit a much stronger correlation in the $H^{0}-126$ case, mostly due to the the $\Delta \rho$ constraints, as shown in the left panel of figure 17. Comparing with the $h^{0}$ 126 case, in which $m_{H}$ could be large with a relaxed constraints on $m_{A}$ and $m_{H^{ \pm}}$mass 

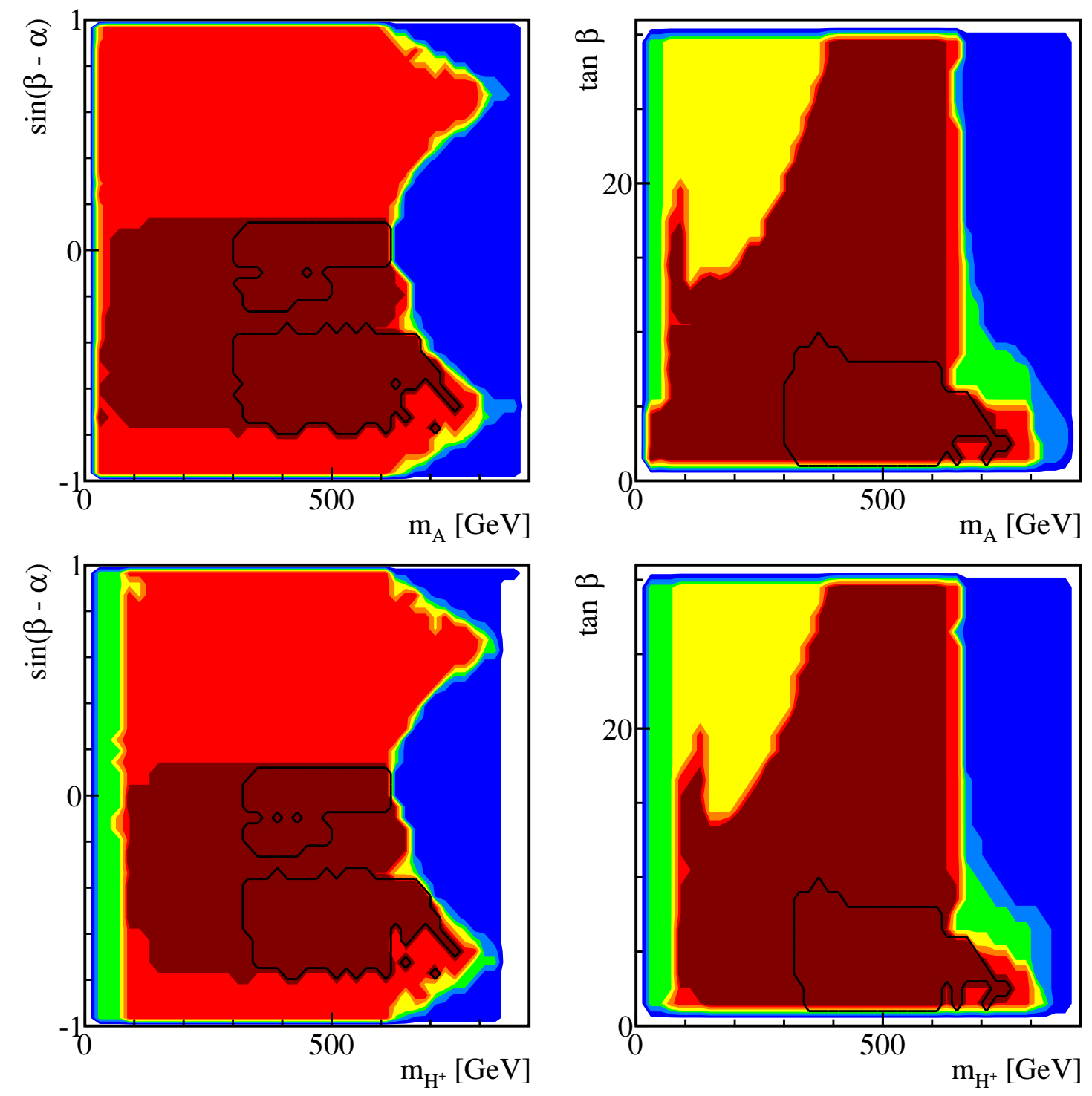

Figure 16. Parameter regions in the $H^{0}-126$ case for $\sin (\beta-\alpha)$ versus $m_{A}$ (upper left panel) and $\tan \beta$ versus $m_{A}$ (upper right panel), as well as similar plots for $m_{H}^{ \pm}$(lower panels). Color coding is the same as figure 14 .

correlation, in the $H^{0}-126$ case, both $m_{h}$ and $m_{H}$ are relatively small. $m_{A}$ and $m_{H^{ \pm}}$should therefore be highly correlated in order to avoid large custodial symmetry breaking in the Higgs sector. However, there is a small strip of allowed region at $m_{H^{ \pm}} \sim 100 \mathrm{GeV}$ with $m_{A}$ between $200-700 \mathrm{GeV}$. This region escapes the $\Delta \rho$ constraint since for $m_{H^{ \pm}} \sim m_{h} \sim m_{H}$, the contribution to $\Delta \rho$ introduced by the large mass difference between $m_{A}$ and $m_{H^{ \pm}}$is cancelled by the $\left(h^{0}, A^{0}\right)$ loop and $\left(H^{0}, A^{0}\right)$ loop. Imposing the flavor constraints again limits $m_{H^{ \pm}}$to be larger than $300 \mathrm{GeV} . m_{A}$ is constrained to be more than $300 \mathrm{GeV}$ as well due to the correlations.

The right panel of figure 17 shows the parameter region of $m_{A}$ versus $m_{h}$, which does not show much correlation. For $m_{h} \lesssim 90 \mathrm{GeV}$, low values of $m_{A} \lesssim 100 \mathrm{GeV}$ is 

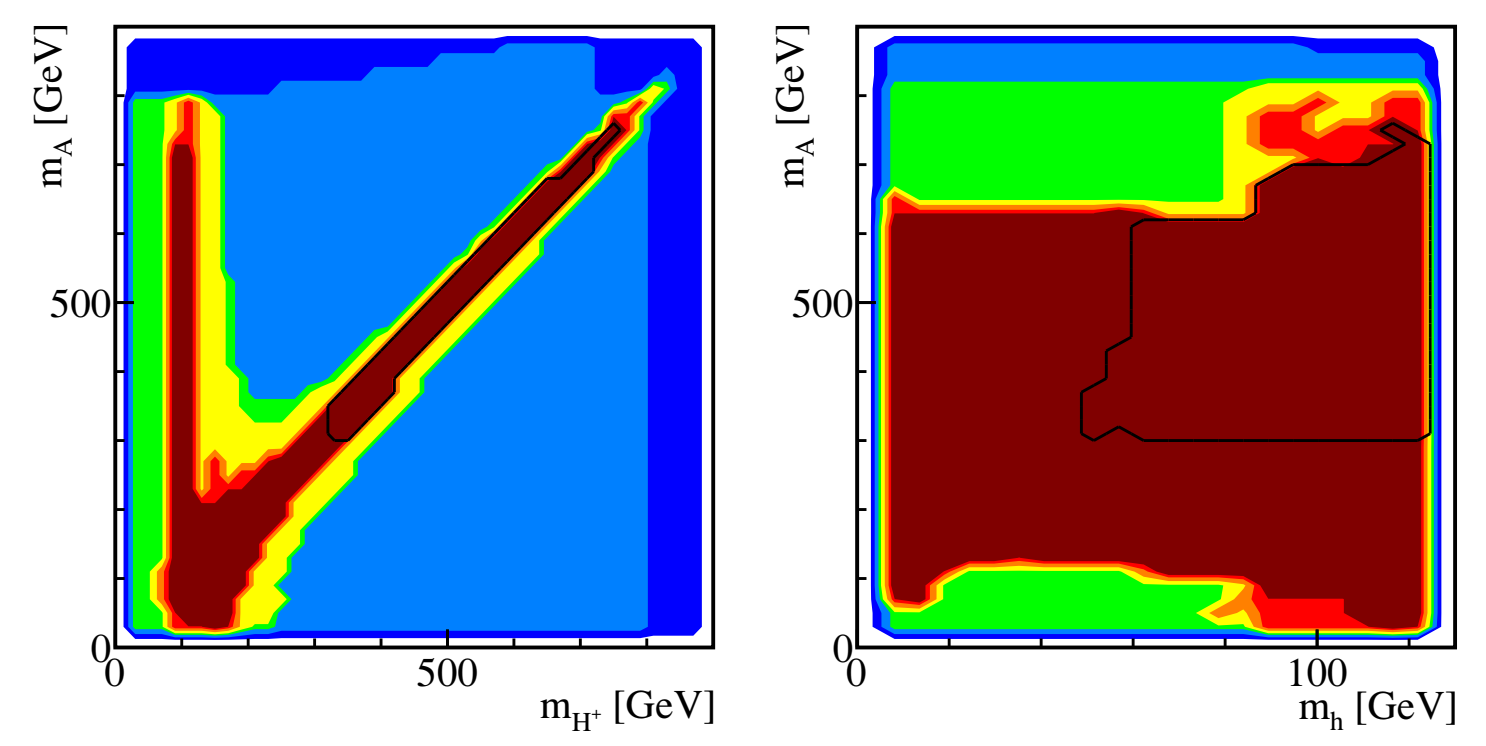

Figure 17. Parameter regions in the $H^{0}-126$ case for $m_{A}$ versus $m_{H^{ \pm}}$(left panel) and $m_{h}$ (right panel). Color coding is the same as figure 14 .

excluded by LEP searches of $h^{0} A^{0}$ channel [53]. High values of $m_{A} \gtrsim 600 \mathrm{GeV}$ are excluded for $m_{h}<90 \mathrm{GeV}$. This is because such a large value of $m_{A}$ can only be realized for $|\sin (\beta-\alpha)|>0.3$ (see the upper-left panel of figure 16). Such regions of $|\sin (\beta-\alpha)|>0.3$ and $m_{h}<90 \mathrm{GeV}$ are excluded by the LEP Higgs search of $h^{0} Z$ channel [54], as shown clearly in the $m_{h}$ versus $\sin (\beta-\alpha)$ plot (left panel of figure 15). Such excluded regions for large $m_{A}$ (and large $m_{H^{ \pm}}$due to correlation) also appears in the $\tan \beta$ versus $m_{A}\left(m_{H^{ \pm}}\right)$ plots in figure 16.

We end the section with the following observations:

- Contrary to the $h^{0}-126$ case, fixing the heavy CP-even Higgses to be the $126 \mathrm{GeV}$ resonance forces us into a small narrow region of $\sin (\alpha-\beta) \sim 0$ with $\tan \beta \lesssim 8$ or an extended region of $-0.8 \lesssim \sin (\alpha-\beta) \lesssim-0.05$ with less restrictions on $\tan \beta$.

- The light CP-even Higgs can have mass of any value up to $126 \mathrm{GeV}$, with smaller $m_{h}$ only allowed for $\sin (\beta-\alpha) \sim 0$. Note that the case of nearly degenerate $h^{0}$ and $H^{0}$ is allowed, as studied in detail in ref. [22].

- $m_{A}$ and $m_{H^{ \pm}}$exhibit a strong correlation: $m_{A} \simeq m_{H^{ \pm}}$, due to $\Delta \rho$ constraints.

- Flavor bounds impose the strong constraints: $\tan \beta \lesssim 10, m_{h}>50 \mathrm{GeV}$, and $m_{H^{ \pm}}>$ $300 \mathrm{GeV} . m_{A}$ is also constrained to be more than $300 \mathrm{GeV}$ due to the correlation between $m_{A}$ and $m_{H^{ \pm}}$.

\section{Other Higgs channels}

Thus far, we have concentrated on the gluon fusion production mechanism and the dominant $\gamma \gamma, Z Z$ and $W W$ decay channels for the Higgs. The vector boson fusion channel is 

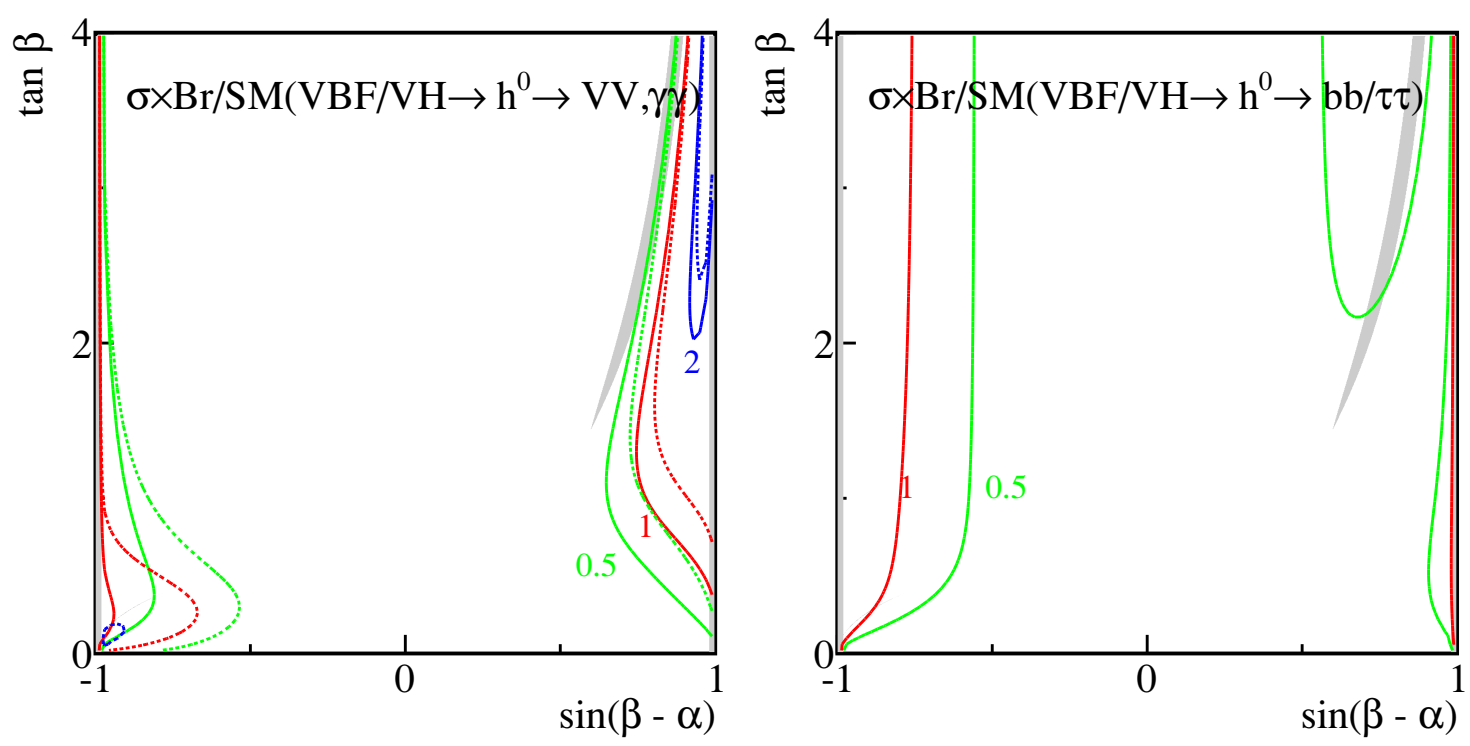

Figure 18. $\sigma \times \mathrm{Br} / \mathrm{SM}$ for $V B F / V H \rightarrow h^{0} \rightarrow W W / Z Z$ (solid curves in left panel), $\gamma \gamma$ (dashed curves in left panel) and $V B F / V H \rightarrow h^{0} \rightarrow b b / \tau \tau$ (right panel) for the $h^{0}-126$ case. The contour lines show $\sigma \times \mathrm{Br} / \mathrm{SM}=0.5$ (green), 1 (red) and 2 (blue). The shaded gray regions correspond to the signal regions where cross sections of $\gamma \gamma$ and $W W / Z Z$ channels satisfy eq. (3.7) as well as $R_{b}$.

another important production channel for the CP-even Higgses. For certain Higgs decay channels, for example, $\tau \tau$ mode, VBF production is the one that provides the dominant sensitivity due to the excellent discrimination of the backgrounds using the two forward tagging jets and the central jet-veto [82]. Other production channels, $V H$ and $t t H$ associated production, can also be of interest for Higgs decay to $b b$. In this section, we discuss the cross sections in other search channels for both $h^{0}$ and $H^{0}$ when they are interpreted as the observed $126 \mathrm{GeV}$ scalar.

In figure 18, we show the normalized cross sections for the $W W / Z Z, \gamma \gamma$ (left panel) and $b b / \tau \tau$ (right panel) final states via VBF or $V H$ associated production (both production cross sections are controlled by $h^{0} V V$ coupling) in the $\tan \beta$ versus $\sin (\beta-\alpha)$ plane for the $h^{0}-126$ case. For $V B F / V H \rightarrow h^{0} \rightarrow W W / Z Z$, both the production and decay are proportional to $\sin (\beta-\alpha)$, resulting in regions highly centered around $\sin (\beta-\alpha) \sim \pm 1$ for any enhancement above the SM value. For the currently preferred gray Higgs signal regions, $V B F / V H \rightarrow h^{0} \rightarrow W W / Z Z$ is typically in the range of $0.5-1$ of the SM value.

The current observation of the Higgs signal has been fitted into the signal strength in both the gluon fusion channel and $\mathrm{VBF}$ channel for $\gamma \gamma, W W$ and $Z Z$ final states [4-7]. Imposing the $95 \%$ C.L. contours of the $\mu_{g g F+t t H} \times \mathrm{B} / \mathrm{B}_{\mathrm{SM}}$ versus $\mu_{V B F+V H} \times \mathrm{B} / \mathrm{B}_{\mathrm{SM}}$ on top of the one-dimensional gluon fusion signal regions as given in eq. (3.7) does not lead to additional reduction of the signal parameter space, given the VBF channel is relatively loosely constrained.

For $V B F / V H \rightarrow h^{0} \rightarrow b b / \tau \tau$, the cross section is suppressed for most of the regions, except in the neighborhood of $\sin (\beta-\alpha)= \pm 1$ where SM rates can be achieved. The current preferred signal regions typically have a suppression of 0.5 or stronger for this 

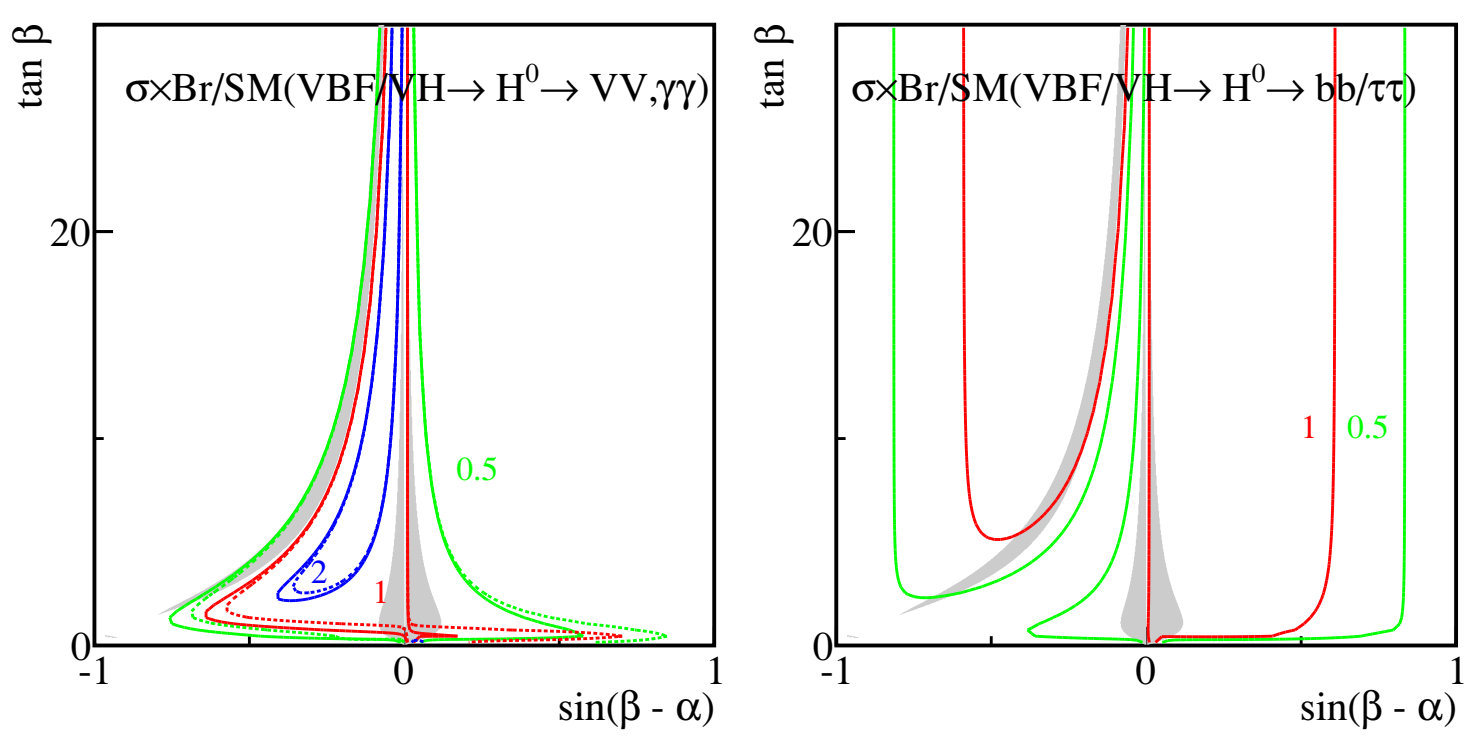

Figure 19. $\sigma \times \mathrm{Br} / \mathrm{SM}$ for $V B F / V H \rightarrow H^{0} \rightarrow W W / Z Z, \gamma \gamma$ (left) and $V B F / V H \rightarrow H^{0} \rightarrow b b / \tau \tau$ (right) for the $H^{0}-126$ case. Color coding is the same as in figure 18.

$b b / \tau \tau$ channel. There is also a strong inverse correlation between the $W W / Z Z$ and $b b / \tau \tau$ channels, since an increase in $b b$ decay branching fraction can only occur at the expense of $W W$. Given the relatively loose bounds on the signal strength in the $b b$ and $\tau \tau$ channels from the LHC and the Tevatron experiments [4, 83-86], imposing the current search results for $b b$ and $\tau \tau$ channels does not lead to further reduction of the signal parameter space.

Figure 19 show the $\sigma \times \mathrm{Br} / \mathrm{SM}$ plots for $V V, \gamma \gamma$, and $b b / \tau \tau$ channel via $\mathrm{VBF} / V H$ production for the $H^{0}-126$ case. The qualitative features of the $V V, \gamma \gamma$ plot is the same as that of figure 12. The currently favored gray signal regions typically correspond to a normalized cross section of $V B F / V H \rightarrow H^{0} \rightarrow W W / Z Z$ around 1 as well.

The $b b / \tau \tau$ channel, however, exhibits a very different behavior. For two regions of $-0.6 \leq \sin (\beta-\alpha) \leq-0.1$ and $0 \leq \sin (\beta-\alpha) \leq 0.6$ (regions enclosed by the red curves in the right panel of figure 19), a normalized cross section of at least the SM signal strength can be achieved. A strong suppression, sometimes as small as 0.1 , can be obtained in the other regions. The currently favored gray signal region near $\sin (\beta-\alpha) \sim 0$ corresponds to $\sigma / \sigma_{\mathrm{SM}}$ of order 1 for $V B F / V H \rightarrow H^{0} \rightarrow b b / \tau \tau$ channel, while a suppression as large as 0.5 is possible for the extended regions in negative $\sin (\beta-\alpha)$. The inverse correlation between $b b / \tau \tau$ and $W W$ channels also appears in the $H^{0}-126$ case. Similar to the $h^{0}-126$ case, imposing the $95 \%$ C.L. range for the VBF process for $\gamma \gamma$ and $W W / Z Z$ channel, as well as the signal strength obtained from the $b b$ and $\tau \tau$ modes does not lead to further reduction of the signal region.

We also studied $g g \rightarrow h^{0}, H^{0} \rightarrow b b / \tau \tau$ channel for both the $h^{0}-126$ and $H^{0}-126$ cases, and noticed that for the currently favored Higgs signal regions, a factor of 2 enhancement could be realized. 


\section{Conclusions}

In this paper, we presented a detailed analysis of the Type II 2HDM (with an imposed $Z_{2}$ symmetry) parameter space, identifying either the light or the heavy CP-even Higgs as the recently discovered resonance at $126 \mathrm{GeV}$. We scanned the remaining five parameters $\sin (\beta-\alpha), \tan \beta, m_{A}, m_{H^{ \pm}}$, and $m_{H}$ or $m_{h}$ while fixing either $m_{h}$ or $m_{H}$ to be $126 \mathrm{GeV}$. We took into account all the theoretical constraints, precision measurements, as well as current experimental search limits on the Higgses. We further studied the implications on the parameter space once flavor constraints are imposed. We found unique features in each of these two cases.

In the $h^{0}-126$ case, we are forced into regions of parameter space where $\sin (\beta-\alpha)= \pm 1$ with $\tan \beta$ between 0.5 to 4 , or an extended region of $0.55<\sin (\beta-\alpha)<0.9$, with $\tan \beta$ constrained to be in the range of 1.5 to 4 . There is, however, a wide range of values that are still allowed for the masses of the heavy CP-even, pseudo scalar and charged Higgses. The Higgs masses are typically not correlated, except when $m_{A, H^{ \pm}} \gtrsim 600 \mathrm{GeV}$ and $\sin (\beta-\alpha)>0$ where there is a strong correlation between $m_{A}$ and $m_{H^{ \pm}}$because of the $\Delta \rho$ constraint. Imposing flavor constraints further restricts $m_{H^{ \pm}}>300 \mathrm{GeV}$.

In the $H^{0}-126$ case, we are forced into an orthogonal region of parameter space where $\sin (\beta-\alpha) \sim 0, \tan \beta \lesssim 8$ or an extended region of $-0.8 \lesssim \sin (\alpha-\beta) \lesssim-0.05$ with less restricted $\tan \beta . m_{A}$ and $m_{H^{ \pm}}$exhibit strong correlations: $m_{A} \simeq m_{H^{ \pm}}$, due to the $\Delta \rho$ constraint. The interesting scenario of the light CP-even Higgs being close to $126 \mathrm{GeV}$ still survives. Imposing flavor bounds further shrinks the parameter space considerably: $\tan \beta \lesssim 10, m_{h}>50 \mathrm{GeV}, m_{H^{ \pm}}>300 \mathrm{GeV}$, and $m_{A}>300 \mathrm{GeV}$.

Note that in both cases, the extended region in $\sin (\beta-\alpha)$ is of particular interest, since a deviation of the Higgs coupling to $W W$ and $Z Z$ can be accommodated for the observed Higgs signal at $126 \mathrm{GeV}$.

We find that in either of these scenarios, one can identify regions of parameter space that pass all theoretical and experimental bounds and still allow a slightly higher than SM rate to diphotons. $\gamma \gamma$ and $W W / Z Z$ rates are most likely strongly correlated: $\gamma \gamma: V V \sim 1$ for the normalized cross sections.

We further studied the implication for the Higgs production via VBF or $V H$ process, and decays to $b b, \tau \tau$ channels. We found that in the $h^{0}-126$ case, both $V B F / V H \rightarrow$ $h^{0} \rightarrow b b / \tau \tau, W W / Z Z$ could be significantly suppressed in the Higgs signal region. For the $H^{0}-126$ case, $V B F / V H \rightarrow H^{0} \rightarrow W W / Z Z$ channel is almost the SM strength. Possible suppression of $b b / \tau \tau$ channel up to 0.5 is possible for the extended signal regions in negative $\sin (\beta-\alpha)$. Future observation of the $b b$ and $\tau \tau$ modes can provide valuable information for the parameter regions of the type II $2 \mathrm{HDM}$.

Comparing to the MSSM, with its Higgs sector being a restricted type II 2HDM and the tree level Higgs spectrum completely determined by $m_{A}$ and $\tan \beta$, the parameter regions of the general Type II $2 \mathrm{HDM}$ is much more relaxed. Unlike the MSSM in which the $h^{0}-126$ case corresponds to the decoupling region where $m_{A} \gtrsim 300 \mathrm{GeV}$, and the $H^{0}$ $126 \mathrm{GeV}$ case corresponds to the non-decoupling region where $m_{A} \sim 100-130 \mathrm{GeV}$ [87], the value of $m_{A}$ in the general Type II $2 \mathrm{HDM}$ could vary over the entire viable region up 
to about $800 \mathrm{GeV}$. The MSSM relation of $m_{A} \sim m_{H^{ \pm}} \sim m_{H}$ in the decoupling region is also much more relaxed in the Type II 2HDM. No obvious correlation is observed between $m_{A}, m_{H^{ \pm}}$, and $m_{H}$ for the $h^{0}-126$ case, except for the region with large $m_{A, H^{ \pm}} \gtrsim 600 \mathrm{GeV}$. Note also that in the Type II $2 \mathrm{HDM}$ with $Z_{2}$ symmetry (such that $m_{12}=0$ ) that we are considering, with the additional perturbativity and unitarity constraints imposed, there is an upper limit of about $800 \mathrm{GeV}$ for the mass of $H^{0}, A^{0}$ and $H^{ \pm}$. The presence of an upper bound on the heavy Higgs masses reiterates our point that unlike the MSSM, there is no sensible decoupling limit in this case where only one light SM-like Higgs appears in the low energy spectrum with other Higgses heavy and decouple.

Observations of extra Higgses in the future would further pin down the Higgs sector beyond the SM. While the conventional decay channels of Higgses to SM particles continue to be important channels to search for extra Higgses, novel decay channels of a heavy Higgs into light Higgses or light Higgs plus gauge boson could also appear. Future work along the lines of collider phenomenology of multiple Higgs scenarios is definitely warranted.

\section{Acknowledgments}

We thank L. Carpenter for her participation at the beginning of this project. We would also like to thank David Lopez-Val for useful discussions and Oscar Stål for sharing the 2HDMC package. This work was supported by the Department of Energy under Grant DEFG02-04ER-41298.

Open Access. This article is distributed under the terms of the Creative Commons Attribution License (CC-BY 4.0), which permits any use, distribution and reproduction in any medium, provided the original author(s) and source are credited.

\section{References}

[1] ATLAS collaboration, Observation of a new particle in the search for the Standard Model Higgs boson with the ATLAS detector at the LHC, Phys. Lett. B 716 (2012) 1 [arXiv: 1207.7214] [INSPIRE].

[2] ATLAS collaboration, Combined measurements of the mass and signal strength of the Higgs-like boson with the ATLAS detector using up to $25 \mathrm{fb}^{-1}$ of proton-proton collision data, ATLAS-CONF-2013-014, CERN, Geneva Switzerland (2013).

[3] CMS collaboration, Observation of a new boson at a mass of $125 \mathrm{GeV}$ with the CMS experiment at the LHC, Phys. Lett. B 716 (2012) 30 [arXiv:1207.7235] [INSPIRE].

[4] CMS collaboration, Combination of Standard Model Higgs boson searches and measurements of the properties of the new boson with a mass near $125 \mathrm{GeV}$, CMS-PAS-HIG-13-005, CERN, Geneva Switzerland (2013).

[5] ATLAS collaboration, Study of the spin of the new boson with up to $25 \mathrm{fb}^{-1}$ of ATLAS data, ATLAS-CONF-2013-040, CERN, Geneva Switzerland (2013).

[6] ATLAS collaboration, Combined coupling measurements of the Higgs-like boson with the ATLAS detector using up to $25 \mathrm{fb}^{-1}$ of proton-proton collision data, ATLAS-CONF-2013-034, CERN, Geneva Switzerland (2013). 
[7] ATLAS collaboration, Measurements of Higgs boson production and couplings in diboson final states with the ATLAS detector at the LHC, Phys. Lett. B 726 (2013) 88 [arXiv: 1307.1427] [INSPIRE].

[8] G. Branco et al., Theory and phenomenology of two-Higgs-doublet models, Phys. Rept. 516 (2012) 1 [arXiv:1106.0034] [inSPIRE].

[9] H. Haber, G.L. Kane and T. Sterling, The Fermion mass scale and possible effects of Higgs bosons on experimental observables, Nucl. Phys. B 161 (1979) 493 [INSPIRE].

[10] L.J. Hall and M.B. Wise, Flavor changing Higgs-boson couplings, Nucl. Phys. B 187 (1981) 397 [INSPIRE].

[11] J.F. Donoghue and L.F. Li, Properties of charged Higgs bosons, Phys. Rev. D 19 (1979) 945 [INSPIRE].

[12] P. Ferreira, R. Santos, M. Sher and J.P. Silva, Implications of the LHC two-photon signal for two-Higgs-doublet models, Phys. Rev. D 85 (2012) 077703 [arXiv:1112.3277] [InSPIRE].

[13] P. Ferreira, R. Santos, M. Sher and J.P. Silva, Could the LHC two-photon signal correspond to the heavier scalar in two-Higgs-doublet models?, Phys. Rev. D 85 (2012) 035020 [arXiv: 1201.0019] [INSPIRE].

[14] H. Cheon and S.K. Kang, Constraining parameter space in type-II two-Higgs doublet model in light of a $126 \mathrm{GeV}$ Higgs boson, JHEP 09 (2013) 085 [arXiv: 1207.1083] [INSPIRE].

[15] A. Drozd, B. Grzadkowski, J.F. Gunion and Y. Jiang, Two-Higgs-doublet models and enhanced rates for a $125 \mathrm{GeV}$ Higgs, JHEP 05 (2013) 072 [arXiv: 1211.3580] [INSPIRE].

[16] S. Chang et al., Comprehensive study of two Higgs doublet model in light of the new boson with mass around $125 \mathrm{GeV}$, JHEP 05 (2013) 075 [arXiv: 1210.3439] [INSPIRE].

[17] C.-Y. Chen and S. Dawson, Exploring two Higgs doublet models through Higgs production, Phys. Rev. D 87 (2013) 055016 [arXiv:1301.0309] [INSPIRE].

[18] B. Grinstein and P. Uttayarat, Carving out parameter space in type-II two Higgs doublets model, JHEP 06 (2013) 094 [Erratum ibid. 09 (2013) 110] [arXiv: 1304.0028] [INSPIRE].

[19] C.-W. Chiang and K. Yagyu, Implications of Higgs boson search data on the two-Higgs doublet models with a softly broken $Z_{2}$ symmetry, JHEP 07 (2013) 160 [arXiv:1303.0168] [INSPIRE].

[20] N. Craig and S. Thomas, Exclusive signals of an extended Higgs sector, JHEP 11 (2012) 083 [arXiv: 1207.4835] [INSPIRE].

[21] L. Basso et al., Probing the charged Higgs boson at the LHC in the CP-violating type-II 2HDM, JHEP 11 (2012) 011 [arXiv: 1205.6569] [INSPIRE].

[22] P. Ferreira, R. Santos, H.E. Haber and J.P. Silva, Mass-degenerate Higgs bosons at $125 \mathrm{GeV}$ in the two-Higgs-doublet model, Phys. Rev. D 87 (2013) 055009 [arXiv:1211.3131] [INSPIRE].

[23] G. Burdman, C.E. Haluch and R.D. Matheus, Is the LHC observing the pseudo-scalar state of a two-Higgs doublet model?, Phys. Rev. D 85 (2012) 095016 [arXiv:1112.3961] [INSPIRE].

[24] E. Cervero and J.-M. Gerard, Minimal violation of flavour and custodial symmetries in a vectophobic two-Higgs-doublet-model, Phys. Lett. B 712 (2012) 255 [arXiv:1202.1973] [INSPIRE]. 
[25] J. Shu and Y. Zhang, Impact of a CP-violating Higgs: from LHC to baryogenesis, Phys. Rev. Lett. 111 (2013) 091801 [arXiv: 1304.0773] [INSPIRE].

[26] B. Coleppa, K. Kumar and H.E. Logan, Can the $126 \mathrm{GeV}$ boson be a pseudoscalar?, Phys. Rev. D 86 (2012) 075022 [arXiv: 1208.2692] [INSPIRE].

[27] S. Davidson and H.E. Haber, Basis-independent methods for the two-Higgs-doublet model, Phys. Rev. D 72 (2005) 035004 [Erratum ibid. D 72 (2005) 099902] [hep-ph/0504050] [INSPIRE].

[28] H.E. Haber and D. O'Neil, Basis-independent methods for the two-Higgs-doublet model. II. The significance of $\tan \beta$, Phys. Rev. D 74 (2006) 015018 [hep-ph/0602242] [INSPIRE].

[29] I.F. Ginzburg and M. Krawczyk, Symmetries of two Higgs doublet model and CP-violation, Phys. Rev. D 72 (2005) 115013 [hep-ph/0408011] [INSPIRE].

[30] D. Eriksson, J. Rathsman and O. Stal, 2HDMC: two-Higgs-doublet model calculator physics and manual, Comput. Phys. Commun. 181 (2010) 189 [arXiv:0902.0851] [INSPIRE].

[31] P. Bechtle, O. Brein, S. Heinemeyer, G. Weiglein and K. Williams, New HiggsBounds from LEP and the Tevatron, AIP Conf. Proc. 1200 (2010) 510 [arXiv:0909.4664] [InSPIRE].

[32] P. Bechtle, O. Brein, S. Heinemeyer, G. Weiglein and K.E. Williams, Introducing HiggsBounds 2.0.0, PoS (CHARGED 2010) 027 [arXiv: 1012.5170] [INSPIRE].

[33] P. Bechtle, O. Brein, S. Heinemeyer, G. Weiglein and K.E. Williams, HiggsBounds: confronting arbitrary Higgs sectors with exclusion bounds from LEP and the Tevatron, Comput. Phys. Commun. 181 (2010) 138 [arXiv:0811.4169] [INSPIRE].

[34] P. Bechtle, O. Brein, S. Heinemeyer, G. Weiglein and K.E. Williams, HiggsBounds 2.0.0: confronting neutral and charged Higgs sector predictions with exclusion bounds from LEP and the Tevatron, Comput. Phys. Commun. 182 (2011) 2605 [arXiv:1102.1898] [INSPIRE].

[35] P. Bechtle et al., Recent developments in HiggsBounds and a preview of HiggsSignals, PoS (CHARGED 2012) 024 [arXiv: 1301.2345] [INSPIRE].

[36] N.G. Deshpande and E. Ma, Pattern of symmetry breaking with two Higgs doublets, Phys. Rev. D 18 (1978) 2574 [INSPIRE].

[37] M. Sher, Electroweak Higgs potentials and vacuum stability, Phys. Rept. 179 (1989) 273 [INSPIRE].

[38] A.W. El Kaffas, W. Khater, O.M. Ogreid and P. Osland, Consistency of the two Higgs doublet model and CP-violation in top production at the LHC, Nucl. Phys. B 775 (2007) 45 [hep-ph/0605142] [INSPIRE].

[39] J. Bijnens, J. Lu and J. Rathsman, Constraining general two Higgs doublet models by the evolution of Yukawa couplings, JHEP 05 (2012) 118 [arXiv:1111.5760] [INSPIRE].

[40] I. Ginzburg and I. Ivanov, Tree-level unitarity constraints in the most general 2HDM, Phys. Rev. D 72 (2005) 115010 [hep-ph/0508020] [INSPIRE].

[41] ATLAS collaboration, Measurements of the properties of the Higgs-like boson in the $W W^{(*)} \rightarrow \ell \nu \ell \nu$ decay channel with the ATLAS detector using $25 \mathrm{fb}^{-1}$ of proton-proton collision data, ATLAS-CONF-2013-030, CERN, Geneva Switzerland (2013).

[42] ATLAS collaboration, Measurements of the properties of the Higgs-like boson in the two photon decay channel with the ATLAS detector using $25 \mathrm{fb}^{-1}$ of proton-proton collision data, ATLAS-CONF-2013-012, CERN, Geneva Switzerland (2013). 
[43] ATLAS collaboration, Measurements of the properties of the Higgs-like boson in the four lepton decay channel with the ATLAS detector using $25 \mathrm{fb}^{-1}$ of proton-proton collision data, ATLAS-CONF-2013-013, CERN, Geneva Switzerland (2013).

[44] ATLAS collaboration, Search for the Standard Model Higgs boson produced in association with top quarks in proton-proton collisions at $\sqrt{s}=7 \mathrm{TeV}$ using the ATLAS detector, ATLAS-CONF-2012-135, CERN, Geneva Switzerland (2012).

[45] ATLAS collaboration, Search for the Standard Model Higgs boson produced in association with a vector boson and decaying to a b-quark pair with the ATLAS detector, Phys. Lett. B 718 (2012) 369 [arXiv: 1207.0210] [INSPIRE].

[46] ATLAS collaboration, Search for the Standard Model Higgs boson in the $H \rightarrow \tau^{+} \tau^{-}$decay mode in $\sqrt{s}=7 \mathrm{TeV}$ pp collisions with ATLAS, JHEP 09 (2012) 070 [arXiv:1206.5971] [INSPIRE].

[47] CMS collaboration, Updated measurements of the Higgs boson at $125 \mathrm{GeV}$ in the two photon decay channel, CMS-PAS-HIG-13-001, CERN, Geneva Switzerland (2013).

[48] CMS collaboration, Properties of the Higgs-like boson in the decay $H \rightarrow Z Z \rightarrow 4 \ell$ in pp collisions at $\sqrt{s}=7$ and 8 TeV, CMS-PAS-HIG-13-002, CERN, Geneva Switzerland (2013).

[49] CMS collaboration, Evidence for a particle decaying to $W^{+} W^{-}$in the fully leptonic final state in a Standard Model Higgs boson search in pp collisions at the LHC, CMS-PAS-HIG-13-003, CERN, Geneva Switzerland (2013).

[50] CMS collaboration, Search for the Standard Model Higgs boson decaying to $\tau$ pairs in proton-proton collisions at $\sqrt{s}=7$ and $8 \mathrm{TeV}$, CMS-PAS-HIG-13-004, CERN, Geneva Switzerland (2013).

[51] CMS collaboration, Search for Higgs boson production in association with top quark pairs in pp collisions, CMS-PAS-HIG-12-025, CERN, Geneva Switzerland (2012).

[52] CMS collaboration, Search for the Standard Model Higgs boson produced in association with $W$ or $Z$ bosons, and decaying to bottom quarks for HCP 2012, CMS-PAS-HIG-12-044, CERN, Geneva Switzerland (2012).

[53] ALEPH, DELPHI, L3, OPAL and LEP Working Group for Higgs Boson SEARCHES collaborations, S. Schael et al., Search for neutral MSSM Higgs bosons at LEP, Eur. Phys. J. C 47 (2006) 547 [hep-ex/0602042] [InSPIRE].

[54] LEP Working Group for Higgs boson searches, ALEPH, DELPHI, L3 and OPAL collaborations, R. Barate et al., Search for the Standard Model Higgs boson at LEP, Phys. Lett. B 565 (2003) 61 [hep-ex/0306033] [INSPIRE].

[55] LeP Higgs Working Group for Higgs boson searches, AlEPH, DELPHI, L3 and OPAL collaborations, Search for charged Higgs bosons: preliminary combined results using LEP data collected at energies up to $209 \mathrm{GeV}$, hep-ex/0107031 [INSPIRE].

[56] ALEPH collaboration, A. Heister et al., Search for charged Higgs bosons in $e^{+} e^{-}$collisions at energies up to $\sqrt{s}=209 \mathrm{GeV}$, Phys. Lett. B 543 (2002) 1 [hep-ex/0207054] [INSPIRE].

[57] Tevatron New Physics Higgs Working Group, CDF and D0 collaborations, Updated combination of CDF and D0 searches for Standard Model Higgs boson production with up to $10.0 \mathrm{fb}^{-1}$ of data, arXiv: 1207.0449 [INSPIRE].

[58] ATLAS collaboration, Search for neutral MSSM Higgs bosons in $\sqrt{s}=7 \mathrm{TeV}$ pp collisions at ATLAS, ATLAS-CONF-2012-094, CERN, Geneva Switzerland (2012). 
[59] ATLAS collaboration, Search for charged Higgs bosons decaying via $H^{+} \rightarrow \tau \nu$ in top quark pair events using pp collision data at $\sqrt{s}=7 \mathrm{TeV}$ with the ATLAS detector, JHEP 06 (2012) 039 [arXiv:1204.2760] [INSPIRE].

[60] ATLAS collaboration, Search for a light charged Higgs boson in the decay channel $H^{+} \rightarrow c \bar{s}$ in $t \bar{t}$ events using pp collisions at $\sqrt{s}=7 \mathrm{TeV}$ with the ATLAS detector, Eur. Phys. J. C $7 \mathbf{3}$ (2013) 2465 [arXiv: 1302.3694] [INSPIRE].

[61] CMS collaboration, Search for neutral Higgs bosons decaying to $\tau$ pairs in pp collisions at $\sqrt{s}=7 \mathrm{TeV}$, Phys. Lett. B 713 (2012) 68 [arXiv:1202.4083] [INSPIRE].

[62] CMS collaboration, Search for MSSM neutral Higgs bosons decaying to $\tau$ pairs in pp collisions, CMS-PAS-HIG-12-050, CERN, Geneva Switzerland (2012).

[63] CMS collaboration, Search for a Higgs boson decaying into a b-quark pair and produced in association with b-quarks in proton-proton collisions at 7 TeV, Phys. Lett. B 722 (2013) 207 [arXiv: 1302.2892] [INSPIRE].

[64] CMS collaboration, Search for a light charged Higgs boson in top quark decays in pp collisions at $\sqrt{s}=7 \mathrm{TeV}$, JHEP 07 (2012) 143 [arXiv: 1205.5736] [INSPIRE].

[65] M.E. Peskin and T. Takeuchi, A new constraint on a strongly interacting Higgs sector, Phys. Rev. Lett. 65 (1990) 964 [INSPIRE].

[66] J.F. Gunion, H.E. Haber, G. Kane and S. Dawson, The Higgs hunter's guide, Addison-Wesley Publishing Company, U.S.A. (1990) [INSPIRE].

[67] Particle Data Group collaboration, K. Nakamura et al., Review of particle physics, J. Phys. G 37 (2010) 075021 [inSPIRE].

[68] H.E. Logan, Radiative corrections to the Z Zb̄ vertex and constraints on extended Higgs sectors, hep-ph/9906332 [INSPIRE].

[69] ALEPH, CDF, D0, DELPHI, L3, OPAL, SLD, LEP Electroweak Working Group, Tevatron Electroweak Working Group and SLD Electroweak and Heavy Flavour Groups collaborations, Precision electroweak measurements and constraints on the Standard Model, arXiv:1012.2367 [INSPIRE].

[70] F. Mahmoudi, SuperIso v2.3: a program for calculating flavor physics observables in supersymmetry, Comput. Phys. Commun. 180 (2009) 1579 [arXiv:0808.3144] [INSPIRE].

[71] Particle Data Group collaboration, J. Beringer et al., Review of particle physics (RPP), Phys. Rev. D 86 (2012) 010001 [inSPIRE].

[72] Heavy Flavor Averaging Group collaboration, Y. Amhis et al., Averages of B-hadron, C-hadron and $\tau$-lepton properties as of early 2012, arXiv:1207.1158 [INSPIRE].

[73] Heavy Flavor Averaging Group online updates webpage, http://www.slac.stanford.edu/xorg/hfag.

[74] Belle collaboration, I. Adachi et al., Measurement of $B^{-} \rightarrow \tau^{-} \bar{\nu}_{\tau}$ with a hadronic tagging method using the full data sample of Belle, Phys. Rev. Lett. 110 (2013) 131801 [arXiv: 1208.4678] [INSPIRE].

[75] BABAR collaboration, P. del Amo Sanchez et al., Measurement of the absolute branching fractions for $D_{s}^{-} \rightarrow \ell^{-} \bar{\nu}_{\ell}$ and extraction of the decay constant $f_{D_{s}}$, Phys. Rev. D 82 (2010) 091103 [arXiv: 1008.4080] [INSPIRE]. 
[76] LHCb collaboration, Measurement of the $B_{s}^{0} \rightarrow \mu^{+} \mu^{-}$branching fraction and search for $B^{0} \rightarrow \mu^{+} \mu^{-}$decays at the LHCb experiment, Phys. Rev. Lett. 111 (2013) 101805 [arXiv: 1307.5024] [INSPIRE].

[77] BABAr collaboration, B. Aubert et al., A search for the rare decay $B^{0} \rightarrow \tau^{+} \tau^{-}$at BABAR, Phys. Rev. Lett. 96 (2006) 241802 [hep-ex/0511015] [INSPIRE].

[78] F. Mahmoudi and O. Stal, Flavor constraints on the two-Higgs-doublet model with general Yukawa couplings, Phys. Rev. D 81 (2010) 035016 [arXiv:0907.1791] [INSPIRE].

[79] BABAR collaboration, J. Lees et al., Evidence for an excess of $\bar{B} \rightarrow D^{(*)} \tau^{-} \bar{\nu}_{\tau}$ decays, Phys. Rev. Lett. 109 (2012) 101802 [arXiv:1205.5442] [INSPIRE].

[80] M. Tanaka and R. Watanabe, Tau longitudinal polarization in $B \rightarrow D \tau \nu$ and its role in the search for charged Higgs boson, Phys. Rev. D 82 (2010) 034027 [arXiv:1005.4306] [INSPIRE].

[81] V.D. Barger, J. Hewett and R. Phillips, New constraints on the charged Higgs sector in two Higgs doublet models, Phys. Rev. D 41 (1990) 3421 [INSPIRE].

[82] D.L. Rainwater, D. Zeppenfeld and K. Hagiwara, Searching for $H \rightarrow \tau^{+} \tau^{-}$in weak boson fusion at the CERN LHC, Phys. Rev. D 59 (1998) 014037 [hep-ph/9808468] [INSPIRE].

[83] CMS collaboration, Search for Higgs boson production in association with a top-quark pair and decaying to bottom quarks or tau leptons, CMS-PAS-HIG-13-019, CERN, Geneva Switzerland (2013).

[84] ATLAS collaboration, Search for the bb decay of the Standard Model Higgs boson in associated $W / Z H$ production with the ATLAS detector, ATLAS-CONF-2013-079, CERN, Geneva Switzerland (2013).

[85] ATLAS collaboration, Search for the Standard Model Higgs boson produced in association with top quarks in proton-proton collisions at $\sqrt{s}=7 \mathrm{TeV}$ using the ATLAS detector, ATLAS-CONF-2012-135, CERN, Geneva Switzerland (2012).

[86] CDF and D0 collaboration, T. Aaltonen et al., Higgs boson studies at the Tevatron, Phys. Rev. D 88 (2013) 052014 [arXiv: 1303.6346] [INSPIRE].

[87] N.D. Christensen, T. Han and S. Su, MSSM Higgs bosons at the LHC, Phys. Rev. D 85 (2012) 115018 [arXiv:1203.3207] [inSPIRE]. 